\title{
Discrete Adjoint-Based Design Optimization of Unsteady Turbulent Flows on Dynamic Unstructured Grids
}

\author{
Eric J. Nielsen* \\ NASA Langley Research Center, Hampton, Virginia 23681 \\ Boris Diskin ${ }^{\dagger}$ \\ National Institute of Aerospace, Hampton, Virginia 23666
}

Nail K. Yamaleev ${ }^{*}$

North Carolina A\&T State University, Greensboro, North Carolina 27411

\begin{abstract}
An adjoint-based methodology for design optimization of unsteady turbulent flows on dynamic unstructured grids is described. The implementation relies on an existing unsteady three-dimensional unstructured grid solver capable of dynamic mesh simulations and discrete adjoint capabilities previously developed for steady flows. The discrete equations for the primal and adjoint systems are presented for the backward-difference family of timeintegration schemes on both static and dynamic grids. The consistency of sensitivity derivatives is established via comparisons with complex-variable computations. The current work is believed to be the first verified implementation of an adjoint-based optimization methodology for the true time-dependent formulation of the Navier-Stokes equations in a practical computational code. Large-scale shape optimizations are demonstrated for turbulent flows over a tiltrotor geometry and a simulated aeroelastic motion of a fighter jet.
\end{abstract}

$\begin{array}{ll}a, b, c, d \text { Temporal coefficients } \\ C & \text { Aerodynamic coefficient } \\ C_{T} & \text { Rotor thrust coefficient } \\ \mathbf{D} & \text { Vector of design variables } \\ E & \text { Total energy per unit volume } \\ \mathbf{F} & \text { Flux vector } \\ \mathbf{F}_{i}, \mathbf{F}_{v} & \text { Inviscid and viscous flux vectors } \\ f & \text { Cost function } \\ f, s & \text { General functions } \\ \mathbf{G} & \text { Grid operator } \\ i & \sqrt{-1} \\ i, j, k, n & \text { Indices } \\ i n & \text { Quantity at initial conditions } \\ J & \text { Number of cost function components } \\ \mathcal{K} & m_{x} \times m_{x} \text { linear elasticity coefficient matrix } \\ L & \text { Lagrangian function } \\ m_{x} & \text { Size of vector } \mathbf{X} \\ m_{q} & \text { Size of vector } \mathbf{Q} \\ N & \text { Number of time levels } \\ \hat{\mathbf{n}} & \text { Outward-pointing normal vector } \\ p & \text { Pressure, also cost function exponent } \\ \mathbf{Q} & m_{q} \times 1 \text { vector of volume-averaged } \\ & \text { conserved variables } \\ \mathbf{q} & m_{q} \times 1 \text { vector of conserved variables } \\ \mathbf{R} & m_{q} \times 1 \text { vector of spatial undivided residuals } \\ & \end{array}$

\section{Nomenclature}

$\mathcal{R} \quad m_{x} \times m_{x}$ block diagonal rotation matrix

$\mathcal{R} \quad 3 \times 3$ rotation matrix

$\boldsymbol{\mathcal { R }}_{G C L} \quad m_{q} \times m_{q}$ diagonal GCL residual matrix

$S \quad$ Control volume surface area

$\mathcal{T} \quad 4 \times 4$ transform matrix

$t \quad$ Time

$u, v, w \quad$ Cartesian components of velocity

$V \quad$ Control volume

$\mathcal{V} \quad m_{q} \times m_{q}$ diagonal matrix of cell volumes

W $\quad 3 \times 1$ face velocity vector

$\mathbf{X} \quad m_{x} \times 1$ vector of grid coordinates

$\mathbf{x} \quad 3 \times 1$ position vector

$x \quad$ Independent variable

$x, y, z \quad$ Cartesian coordinate directions

$\varepsilon \quad$ Perturbation

$\Lambda_{f} \quad m_{q} \times 1$ flowfield adjoint variable

$\Lambda_{g} \quad m_{x} \times 1$ grid adjoint variable

$\Theta \quad$ Rotor blade collective setting

$\rho \quad$ Density

$\tau \quad 3 \times 1$ translation vector

$\tau \quad m_{x} \times 1$ translation vector

$\omega \quad$ Cost function component weight

$\Psi \quad$ Rotor azimuth

$\infty \quad$ Quantity at freestream conditions

$\mathbf{R} \quad m_{q} \times 1$ vector of spatial undivided residuals $* \quad$ Target quantity

\footnotetext{
* Research Scientist, Computational AeroSciences Branch, Senior Member AIAA.

$\dagger$ Associate Fellow, also Visiting Associate Professor, Department of Mechanical and Aerospace Engineering, University of Virginia, Member AIAA.

*Associate Professor, Department of Mathematics, Member AIAA.
} 


\section{Introduction}

As computational fluid dynamics (CFD) tools become more efficient, accurate, and robust, their role in the analysis and design of new aerospace configurations continues to increase. Computational methods have already become a major integrated component of industrial practices. The use of CFD has been traditionally confined to the steady regime; however, with recent algorithmic improvements and the persistent growth of computational power, CFD methods have begun to make substantial in-roads in simulating unsteady flow phenomena. Target applications for these methods are widely abundant; typical examples might include the prediction of aeroelastic characteristics, maneuvering flight conditions, six degree-of-freedom simulations, specified motion problems, or flow control simulations, among many others.

In recent years, steady-state CFD methods have been targeted for use in automated design optimization frameworks. In gradient-based design approaches, one of the major challenges is to obtain sensitivity information for the flowfield at a reasonable cost. Conventional black-box finite difference methods ${ }^{[1]}$ suffer from well-known step-size limitations and incur a computational expense that grows linearly with the number of design variables. Forward, or direct, differentiation methods ${ }^{[2]}$ and techniques based on the use of complex variables ${ }^{[3]}$ mitigate the step-size limitation but still suffer from excessive cost in the presence of many design variables, as is often the case with aerodynamic design applications.

Adjoint methods provide a powerful alternative for aerodynamic sensitivity analysis. In this approach, the sensitivities of an objection function are determined through the solution of an auxiliary, or adjoint, set of equations. Adjoint methods may be further categorized into either continuous or discrete approaches, depending on the order in which the governing equations are differentiated and discretized. The discrete approach allows one to account for mesh variation as well; a second adjoint system can be solved to linearize the relationship between the design variables and the mesh operator as described in Ref. [4]. The principal advantage of the adjoint approach is that the computational cost is independent of the number of design variables; a rigorous sensitivity analysis for hundreds of variables can be performed at a cost equivalent to the solution of the governing equations themselves. For examples of the use of such methods, see the references cited in Ref. [5].

The role of adjoint-based methodologies in mesh adaptation strategies should also be noted. Whereas many traditional mesh adaptation schemes rely on heuristic connections between solution gradient information and local mesh spacing requirements, the adjoint equations establish a rigorous mathematical connection between solution accuracy and the computational grid. The approach has proven quite powerful and has enjoyed success where traditional feature-based approaches have consistently failed. Ref. [6] provides a review of recent applications and an extensive list of references on the subject.

Some recent examples of adjoint-based strategies for unsteady aerospace applications are given in Refs. [7]-[13]. The goal of the current work is to extend the time-dependent adjoint formulation for static grids introduced in Ref. [13] and the steady-state discrete adjoint capability developed in Refs. [4] and [14]-[18] to the three-dimensional time-dependent Euler and Reynolds-averaged Navier-Stokes equations. The present approach and implementation are valid for unsteady flows on various grids including static grids, dynamic grids undergoing rigid motion, and general morphing grids governed by a mesh deformation scheme based on a linear elasticity analog. The work is believed to be the first verified implementation of an adjoint-based optimization methodology for the true time-dependent formulation of the Navier-Stokes equations in a practical computational code. In the following sections, the unsteady governing equations are presented as well as various mesh motion strategies. This is followed by the derivation of the discrete adjoint equations for the flowfield and mesh, including details concerning their implementation. Examples demonstrating the discrete consistency of the implementation and applications of the design optimization framework to large-scale problems are also shown.

\section{The Flowfield Equations}

\section{Governing Equations and Solution Method}

Using the approach outlined in Ref. [19], the unsteady Euler and Navier-Stokes equations may be written in the following form for both moving and stationary control volumes:

$$
\frac{\partial}{\partial t} \int_{V} \mathbf{q} d V+\oint_{d V}\left(\mathbf{F}_{i}-\mathbf{F}_{v}\right) \cdot \hat{\mathbf{n}} d S=0,
$$


where $V$ is the control volume bounded by the surface $d V$. The vector $\mathbf{q}$ represents the conserved variables for mass, momentum, and energy, and the vectors $\mathbf{F}_{i}$ and $\mathbf{F}_{v}$ denote the inviscid and viscous fluxes, respectively. Note that for a moving control volume, the inviscid flux vector must account for the difference in the fluxes due to the movement of control volume faces. Given a flux vector $\mathbf{F}$ on a static grid, the corresponding flux $\mathbf{F}_{i}$ on a moving grid can be defined as $\mathbf{F}_{i}=\mathbf{F}-\mathbf{q}(\mathbf{W} \cdot \hat{\mathbf{n}})$, where $\mathbf{W}$ is a local face velocity and $\hat{\mathbf{n}}$ is an outward-pointing unit face normal.

By defining a volume-averaged quantity $\mathbf{Q}$ within each control volume,

$$
\mathbf{Q}=\frac{\int_{V} \mathbf{q} d V}{V},
$$

the conservation equations take the form

$$
\frac{\partial(\mathbf{Q} V)}{\partial t}+\oint_{d V}\left(\mathbf{F}_{i}-\mathbf{F}_{v}\right) \cdot \hat{\mathbf{n}} d S=0
$$

where the conserved variables and inviscid flux vectors are defined as $\mathbf{Q}=[\rho, \rho u, \rho v, \rho w, E]^{T}$ and

$$
\mathbf{F}_{i}=\left[\begin{array}{c}
\rho\left(u-W_{x}\right) \\
\rho u\left(u-W_{x}\right)+p \\
\rho v\left(u-W_{x}\right) \\
\rho w\left(u-W_{x}\right) \\
(E+p)\left(u-W_{x}\right)+W_{x} p
\end{array}\right] \hat{\mathbf{i}}+\left[\begin{array}{c}
\rho\left(v-W_{y}\right) \\
\rho u\left(v-W_{y}\right) \\
\rho v\left(v-W_{y}\right)+p \\
\rho w\left(v-W_{y}\right) \\
(E+p)\left(v-W_{y}\right)+W_{y} p
\end{array}\right] \hat{\mathbf{j}}+\left[\begin{array}{c}
\rho\left(w-W_{z}\right) \\
\rho u\left(w-W_{z}\right) \\
\rho v\left(w-W_{z}\right) \\
\rho w\left(w-W_{z}\right)+p \\
(E+p)\left(w-W_{z}\right)+W_{z} p
\end{array}\right] \hat{\mathbf{k}} .
$$

The viscous flux vector $\mathbf{F}_{v}$ is not explicitly shown here. The equations are closed with the perfect gas equation of state and an appropriate turbulence model for the eddy viscosity. Finally, it is worth noting that for the special case of a spatially and temporally constant state vector, e.g., $\mathbf{Q}=(1,0,0,0,0)^{T}$, the conservation equations reduce to the Geometric Conservation Law (GCL) ${ }^{[20]}$ :

$$
\frac{\partial V}{\partial t}=\oint_{d V} \mathbf{W} \cdot \hat{\mathbf{n}} d S
$$

In computational practice, the discrete GCL residual is added to the flow equations to preserve a constant solution on dynamic grids. ${ }^{[19]}$

References [14], [19], [21], and [22] describe the flow solver used in the current work. The code can be used to perform aerodynamic simulations across the speed range and an extensive list of options and solution algorithms is available for spatial and temporal discretizations on general static or dynamic mixedelement unstructured meshes which may or may not contain overset grid topologies.

In the current study, the spatial discretization uses a finite-volume approach, in which the dependent variables are stored at the vertices of tetrahedral meshes. Inviscid fluxes at cell interfaces are computed using the upwind scheme of Roe, ${ }^{[23]}$ and viscous fluxes are formed using an approach equivalent to a finiteelement Galerkin procedure. For dynamic mesh cases, the mesh velocity terms are evaluated using backward differences consistent with the discrete time derivative; this makes the spatial and GCL residuals dependent on grids at previous time levels. The eddy viscosity is modeled using the one-equation approach of Spalart and Allmaras. ${ }^{[24]}$ Massively parallel scalability is achieved through domain decomposition and message passing communication.

An approximate solution of the linear system of equations formed within each time step is obtained through several iterations of a multicolor Gauss-Seidel point-iterative scheme. The turbulence model is integrated all the way to the wall without the use of wall functions and is solved separately from the mean flow equations at each time step with a time integration and linear system solution scheme identical to that employed for the mean flow equations.

\section{The Grid Equations}

The general grid equations can be defined in the form $\mathbf{G}^{n}(\mathbf{X}, \mathbf{D})=0$, where $\mathbf{X}$ is the mesh (meshes at several time levels may be involved), $\mathbf{D}$ is the vector of design variables, and $n$ denotes the time level and indicates that the grid operator may vary in time. The specific formulations for different grid motions are introduced below. 


\section{Grids Undergoing Rigid Motion}

For problems in which rigid mesh motion is required, the motion is generated by a $4 \times 4$ transform matrix, $\mathcal{T}$, as outlined in Ref. [19]. This transform matrix enables general translations and rotations of the grid according to the relation

$$
\mathbf{x}=\mathcal{T}^{0}
$$

which moves a point from an initial position $\mathbf{x}^{0}=\left(x^{0}, y^{0}, z^{0}\right)^{T}$ to its new position $\mathbf{x}=(x, y, z)^{T}$ :

$$
\left[\begin{array}{c}
x \\
y \\
z \\
1
\end{array}\right]=\left[\begin{array}{cccc}
R_{11} & R_{12} & R_{13} & \tau_{x} \\
R_{21} & R_{22} & R_{23} & \tau_{y} \\
R_{31} & R_{32} & R_{33} & \tau_{z} \\
0 & 0 & 0 & 1
\end{array}\right]\left[\begin{array}{c}
x^{0} \\
y^{0} \\
z^{0} \\
1
\end{array}\right] .
$$

In an expanded form, $\mathbf{x}=\mathcal{R} \mathbf{x}^{0}+\tau$. Here, the $3 \times 3$ matrix $\mathcal{R}$ defines a general rotation and the vector $\tau$ specifies a translation. The matrix $\mathcal{T}$ is generally time-dependent. One useful feature of this approach is that multiple transformations telescope via matrix multiplication. This formulation is particularly attractive for composite parent-child body motion, where motion of one body is often specified relative to another. The reader is referred to the discussion in Ref. [19] for more details. For this formulation, the grid operator at time level $n$ is defined as

$$
\mathbf{G}^{n}\left(\mathbf{X}^{n}, \mathbf{X}^{0}, \mathbf{D}\right) \equiv \mathcal{R}^{n} \mathbf{X}^{0}+\tau^{n}-\mathbf{X}^{n},
$$

where $\mathbf{X}^{0}$ and $\mathbf{X}^{n}$ are the grid vectors at the initial and $n^{\text {th }}$ time levels, respectively; $\mathcal{R}^{n}$ is an $m_{x} \times m_{x}$ block-diagonal matrix with $3 \times 3$ blocks representing rotation and $m_{x}$ being the size of vector $\mathbf{X}^{n}$; and $\tau^{n}$ is an $m_{x}$-size translation vector. The matrix $\mathcal{R}^{n}$ and vector $\tau^{n}$ may explicitly depend on $\mathbf{D}$.

\section{Deforming Grids}

The simplest example of a deforming grid simulation is a static grid undergoing deformations as a result of a shape optimization process. In this case, the grid is not time-dependent and is modeled as an elastic medium which obeys the elasticity relations of solid mechanics. An auxiliary system of linear partial differential equations (PDE's) is solved to determine the mesh coordinates after each shape update. Discretization of these PDE's yields a system of equations

$$
\mathcal{K} \mathbf{X}=\mathbf{X}_{\text {surf }}
$$

where $\mathcal{K}$ represents the elasticity coefficient matrix, $\mathbf{X}$ is the vector of grid coordinates being solved for, and $\mathbf{X}_{\text {surf }}$ is the vector of updated surface coordinates, complemented by zeros for all interior coordinates.

The coefficients of the matrix $\mathcal{K}$ depend on the coordinates of the grid. In the approach followed here, the elasticity equations are discretized on the grid corresponding to the initial time level. Thus, the grid at the initial level satisfies the nonlinear equations

$$
\mathcal{K}^{0}\left(\mathbf{X}^{0}, \mathbf{D}\right) \mathbf{X}^{0}=\mathbf{X}_{\text {surf }}^{0} .
$$

The material properties of the system are chosen based on the local cell geometry and proximity to the surface, and the system is solved using a preconditioned GMRES algorithm. For further details on the approach, see Refs. [16], [19], and [25].

For static grid formulations, the only grid operator used at all times is

$$
\mathbf{G}(\mathbf{X}, \mathbf{D}) \equiv \mathbf{X}_{\text {surf }}-\mathcal{K} \mathbf{X},
$$

where $\mathbf{X}_{\text {surf }}$ may explicitly depend on $\mathbf{D}$. There are situations in which time-dependent deforming grids are required, including aeroelastic deflections of the surface, for which the rigid motion as described in the previous section is not valid. Instead, a morphing mesh formulation is used. In this approach, the linear elasticity equations given by Eq. (9) are solved at each time level with the matrix $\mathcal{K}=\mathcal{K}^{0}$ computed at the initial time level and fixed throughout the time evolution; the vector $\mathbf{X}_{\text {surf }}^{n}$ represents the current body positions. For morphing grids, the operator at time level $n$ is defined as

$$
\mathbf{G}^{n}\left(\mathbf{X}^{n}, \mathbf{D}\right) \equiv \mathbf{X}_{\text {surf }}^{n}-\mathcal{K}^{0} \mathbf{X}^{n}
$$

When the surface motion is governed by the rigid motion relations given by Eq. (6), $\mathbf{X}_{\text {surf }}^{n}$ can be further specified as $\mathbf{X}_{\text {surf }}^{n}=\mathcal{R}^{n} \mathbf{X}_{\text {surf }}^{0}+\tau^{n}$. 


\section{Cost Functions}

The steady-state adjoint implementation described in Refs. [4] and [14]-[18] permits multiple objective functions and explicit constraints of the following form, each containing a summation of individual components:

$$
f_{i}=\sum_{j=1}^{J_{i}} \omega_{j}\left(C_{j}-C_{j}^{*}\right)^{p_{j}} .
$$

Here, $\omega_{j}$ represents a user-defined weighting factor; $C_{j}$ is an aerodynamic coefficient such as the total drag or the pressure or viscous contributions to such quantities, the $\left(^{*}\right)$ superscript indicates a user-defined target value of $C_{j}$; and $p_{j}$ is a user-defined exponent chosen so that $f_{i}$ is a convex functional. The user may specify computational boundaries to which each component function applies. The index $i$ indicates a possibility to introduce several different cost functions or constraints, which may be useful if the user desires separate sensitivities, e.g., for lift, drag, pitching moment, etc.

For the unsteady formulation, similar general cost functions $f_{i}^{n}$ are defined at each time level $n$. The integrated cost function $f_{i}$ is defined as a discrete time integral over a certain time interval $\left[t_{i}^{1}, t_{i}^{2}\right]$ :

$$
f_{i}=\sum_{n=N_{i}^{1}}^{N_{i}^{2}} f_{i}^{n} \Delta t
$$

where time levels $N_{i}^{1}$ and $N_{i}^{2}$ correspond to $t_{i}^{1}$ and $t_{i}^{2}$, respectively. The user now supplies time intervals over which the cost functions are to be used.

\section{Derivation of the Time-Dependent Adjoint Equations}

To derive the time-dependent form of the adjoint equations, the methodology developed in Ref. [13] is used. The governing equations given by Eq. (3) are rewritten as

$$
\frac{\partial(\mathbf{Q} V)}{\partial t}+\mathbf{R}=0, \mathbf{R} \equiv \oint_{d V}\left(\mathbf{F}_{i}-\mathbf{F}_{v}\right) \cdot \hat{\mathbf{n}} d S .
$$

Using a first-order backward difference (BDF1) in time, the equations can be evaluated at time level $n$ as follows:

$$
\mathcal{V}^{n} \frac{\mathbf{Q}^{n}-\mathbf{Q}^{n-1}}{\Delta t}+\mathbf{R}^{n}+\mathcal{R}_{G C L}^{n} \mathbf{Q}^{n-1}=0
$$

Here, $\mathcal{V}^{n}$ and $\mathcal{R}_{G C L}^{n}$ are $m_{q} \times m_{q}$ diagonal matrices, $m_{q}$ is the length of vector $\mathbf{Q}^{n}$, the GCL is discretized in a consistent fashion as

$$
\frac{1}{\Delta t}\left(\mathcal{V}^{n}-\mathcal{V}^{n-1}\right)=\mathcal{R}_{G C L}^{n}
$$

and $\mathbf{R}^{n}$ is the spatial undivided residual. Recall that $\mathbf{R}^{n}$ and $\mathcal{R}_{G C L}^{n}$ depend on grids at the current and previous time levels. Note also that although the BDF1 scheme has been shown here for the sake of simplicity, the derivations for higher-order temporal schemes are similar and included as an appendix.

The discrete adjoint-based optimization methodology is based on the method of Lagrange multipliers, which is used to enforce the governing equations as constraints. For the sake of simplicity in the following presentation, a single cost function is assumed and therefore the index $i$ is omitted. For the time-dependent equations, the Lagrangian functional is defined as follows:

$$
\begin{aligned}
L\left(\mathbf{D}, \mathbf{Q}, \mathbf{X}, \boldsymbol{\Lambda}_{f}, \boldsymbol{\Lambda}_{g}\right) & =\sum_{n=1}^{N} f^{n} \Delta t+\sum_{n=1}^{N}\left[\boldsymbol{\Lambda}_{f}^{n}\right]^{T}\left(\mathcal{V}^{n} \frac{\mathbf{Q}^{n}-\mathbf{Q}^{n-1}}{\Delta t}+\mathbf{R}^{n}+\mathcal{R}_{G C L}^{n} \mathbf{Q}^{n-1}\right) \Delta t \\
& +\sum_{n=1}^{N}\left[\boldsymbol{\Lambda}_{g}^{n}\right]^{T} \mathbf{G}^{n} \Delta t+\left(f^{0}+\left[\boldsymbol{\Lambda}_{f}^{0}\right]^{T} \mathbf{R}^{i n}\right) \Delta t+\left[\boldsymbol{\Lambda}_{g}^{0}\right]^{T} \mathbf{G}^{0} \Delta t
\end{aligned}
$$

where $f^{n} \equiv 0$ for $n<N^{1}$ and $n>N^{2} ; \mathbf{G}^{n}=0$ are the grid equations at time level $n ; \boldsymbol{\Lambda}_{f}^{n}$ and $\boldsymbol{\Lambda}_{g}^{n}$ are vectors of Lagrange multipliers associated with the flow and grid equations at time level $n$, respectively; $\mathbf{D}$ is a vector of design variables; and $\mathbf{R}^{i n}=0$ is the initial condition for the flow equations. 
The Lagrangian is differentiated with respect to $\mathbf{D}$, assuming that $f^{n}$ depends on $\mathbf{Q}^{n}, \mathbf{X}^{n}$, and $\mathbf{D}$; $\mathbf{R}^{\text {in }}$ depends on $\mathbf{Q}^{0}, \mathbf{X}^{0}$, and $\mathbf{D} ; \mathbf{R}^{n}$ depends on $\mathbf{Q}^{n}, \mathbf{X}^{n}, \mathbf{X}^{n-1}$, and $\mathbf{D} ;$ and $\mathcal{R}_{G C L}^{n}$ depends on $\mathbf{X}^{n}$, $\mathbf{X}^{n-1}$, and $\mathbf{D}$. Regrouping terms to isolate the coefficients of $\partial \mathbf{Q}^{n} / \partial \mathbf{D}$ and equating the coefficients to zero yields the final form of the adjoint equations for the flowfield:

$$
\begin{gathered}
\frac{1}{\Delta t}\left(\mathcal{V}^{n} \boldsymbol{\Lambda}_{f}^{n}-\mathcal{V}^{n+1} \boldsymbol{\Lambda}_{f}^{n+1}\right)+\left[\frac{\partial \mathbf{R}^{n}}{\partial \mathbf{Q}^{n}}\right]^{T} \boldsymbol{\Lambda}_{f}^{n}+\mathcal{R}_{G C L}^{n+1} \boldsymbol{\Lambda}_{f}^{n+1}=-\left[\frac{\partial f^{n}}{\partial \mathbf{Q}^{n}}\right]^{T}, \text { for } 1 \leq n \leq N \\
-\frac{1}{\Delta t} \mathcal{V}^{1} \boldsymbol{\Lambda}_{f}^{1}+\left[\frac{\partial \mathbf{R}^{i n}}{\partial \mathbf{Q}^{0}}\right]^{T} \boldsymbol{\Lambda}_{f}^{0}+\mathcal{R}_{G C L}^{1} \boldsymbol{\Lambda}_{f}^{1}=-\left[\frac{\partial f^{0}}{\partial \mathbf{Q}^{0}}\right]^{T}, \text { for the initial time level, }
\end{gathered}
$$

where $\Lambda^{N+1}=0$. Collecting the coefficients of $\partial \mathbf{X}^{n} / \partial \mathbf{D}$ and equating them to zero leads to similar adjoint equations for the grid. Assuming that the grid operator at time level $n, \mathbf{G}^{n}$, depends on $\mathbf{X}^{n}, \mathbf{X}^{0}$, and $\mathbf{D}$, the grid adjoint equations are defined as

$$
\begin{gathered}
-\left[\frac{\partial \mathbf{G}^{n}}{\partial \mathbf{X}^{n}}\right]^{T} \boldsymbol{\Lambda}_{g}^{n}=\left[\frac{\partial f}{\partial \mathbf{X}^{n}}\right]^{T}+\left[\frac{\partial \mathcal{V}}{\partial \mathbf{X}^{n}} \frac{\mathbf{Q}^{n}-\mathbf{Q}^{n-1}}{\Delta t}\right]^{T} \boldsymbol{\Lambda}_{f}^{n} \\
+\sum_{k=0}^{1}\left[\frac{\partial \mathbf{R}^{n+k}}{\partial \mathbf{X}^{n}}+\frac{\partial \mathcal{R}_{G C L}^{n+k}}{\partial \mathbf{X}^{n}} \mathbf{Q}^{n+k-1}\right]^{T} \boldsymbol{\Lambda}_{f}^{n+k}, \text { for } 1 \leq n \leq N ; \\
-\left[\frac{\partial \mathbf{G}^{0}}{\partial \mathbf{X}^{0}}\right]^{T} \boldsymbol{\Lambda}_{g}^{0}=\sum_{n=1}^{N}\left[\frac{\partial \mathbf{G}^{n}}{\partial \mathbf{X}^{0}}\right]^{T} \boldsymbol{\Lambda}_{g}^{n}+\left[\frac{\partial f^{0}}{\partial \mathbf{X}^{0}}\right]^{T}+\left[\frac{\partial \mathbf{R}^{i n}}{\partial \mathbf{X}^{0}}\right]^{T} \mathbf{\Lambda}_{f}^{0}+\left[\frac{\partial \mathbf{R}^{1}}{\partial \mathbf{X}^{0}}+\frac{\partial \mathcal{R}_{G C L}^{1}}{\partial \mathbf{X}^{0}} \mathbf{Q}^{0}\right]^{T} \Lambda_{f}^{1} .
\end{gathered}
$$

The specific form of these equations will be discussed in subsequent sections. With the adjoint coefficients satisfying the flowfield and grid adjoint equations, the sensitivity derivatives are calculated as follows:

$$
\begin{gathered}
\frac{d L}{d \mathbf{D}}=\sum_{n=1}^{N}\left(\frac{\partial f^{n}}{\partial \mathbf{D}}+\left[\boldsymbol{\Lambda}_{f}^{n}\right]^{T}\left[\frac{\partial \mathbf{R}^{n}}{\partial \mathbf{D}}+\frac{\partial \mathcal{R}_{G C L}^{n}}{\partial \mathbf{D}} \mathbf{Q}^{n-1}\right]+\left[\boldsymbol{\Lambda}_{g}^{n}\right]^{T} \frac{\partial \mathbf{G}^{n}}{\partial \mathbf{D}}\right) \Delta t \\
+\left(\frac{\partial f^{0}}{\partial \mathbf{D}}+\left[\boldsymbol{\Lambda}_{f}^{0}\right]^{T} \frac{\partial \mathbf{R}^{i n}}{\partial \mathbf{D}}+\left[\boldsymbol{\Lambda}_{g}^{0}\right]^{T} \frac{\partial \mathbf{G}^{0}}{\partial \mathbf{D}}\right) \Delta t .
\end{gathered}
$$

\section{Implementation}

\section{Flowfield Adjoint Equations}

The implementation and solution of Eqs. (19) and (20) are based largely on the steady-state strategies described in Refs. [4] and [14]-[18]. In this manner, a great deal of software development effort is avoided since the steady and unsteady equations share many similar terms, namely the details of the spatial discretization. However, some fundamental differences in the implementation must be addressed for timedependent problems.

\section{Implications of Reverse Time Integration}

While the discrete solution $\mathbf{Q}^{n}$ for Eq. (3) is determined by marching forward in physical time from $n=0$ to $n=N$, due to the nature of the adjoint equations and their boundary conditions, the solution for $\boldsymbol{\Lambda}_{f}^{n}$ must instead be initiated from $n=N$ and proceed backwards in physical time. Since Eqs. (19) and (20) involve the linearizations $\partial \mathbf{R}^{n} / \partial \mathbf{Q}$ and $\partial f^{n} / \partial \mathbf{Q}$, the flow solution $\mathbf{Q}^{n}$ at all time levels must be available during the reverse integration.

In practice, the most straightforward approach to meeting this requirement is to store $\mathbf{Q}^{n}$ to disk for all $n$ during the solution of Eq. (16). In this case, the storage cost is significant, but the primary advantage is ease of implementation. This is the approach used for the current study. For problems where the mesh is changing in time, the grid point coordinates and associated speeds are also stored. While these mesh-related values could be recovered by performing the mesh motion in reverse, ease of the full storage implementation has been favored. 


\section{Solution Strategy}

As described in Ref. [19], each solution vector $\mathbf{Q}^{n}$ is determined through a dual time-stepping procedure. In this approach, a sequence of subiterations is performed within each physical time step. The procedure relies on an approximate linearization of the discrete residual combined with a pseudo-time term to achieve a scheme directly analogous to that used in Ref. [21] for steady flows. The same subiterative strategy is employed for the time-dependent adjoint equations, following an approach similar to that outlined in Ref. [17]. The Jacobian matrix used to relax the adjoint system is constructed once at each time step $n$ based on the value of $\mathbf{Q}^{n}$, and does not change during the subiterative procedure.

A requirement for performing adjoint solutions is that the iteration scheme be linearly stable. It has been observed in some cases, more often for unsteady problems than for steady ones, that linear stability is not satisfactory. Suggested explanations ${ }^{[18],[26],[27]}$ vary from physical instabilities to instabilities of the numerical schemes involved. The generalized conjugate residual (GCR) scheme described in Ref. [28] has been used to wrap the multicolor Gauss-Seidel iteration as well as the temporal subiterative procedure. This approach has been found to work well in stabilizing otherwise problematic iterations.

\section{Data Storage}

For three-dimensional dynamic grid simulations using a one-equation turbulence model, the reverse time integration and solution techniques outlined above require the storage of twelve floating-point variables per grid point at each time step: six flowfield variables, three mesh coordinates, and three mesh velocities. For large-scale problems involving many time steps, this strategy can easily result in a storage requirement on the order of terabytes of disk space. Strategies for circumventing storage limitations have been suggested in the literature, ${ }^{[9],[29],[30]}$ these may be the focus of future investigations once an initial capability has been established.

In the current implementation, each processor is responsible for reading and writing its local solution for the entire time history to a unique file on disk. Since each file may contain several gigabytes of data, requiring several hundred processors to parse sequential-access files at each time step can be very inefficient. For this reason, direct-access files are used so that the file pointer can be immediately placed at the record of interest. It has been found that this approach can decrease the time required for disk I/O by as much as two orders of magnitude on large cases. The use of asynchronous file I/O was also examined, although it is not currently being used.

\section{Grid Adjoint and Sensitivity Equations}

Depending on the nature of the grid operator $\mathbf{G}$ and the design variables $\mathbf{D}$, the grid adjoint and sensitivity equations may need to be solved at each time level $n$, once at $n=0$, or not at all. If solutions at each time step are required, they are performed at the completion of each step of the adjoint solver, rather than subsequently performing additional loops over the entire range of time levels. In this manner, $\mathbf{Q}^{n}$ is the only solution vector which must be stored for all $n$, while $\Lambda_{f}^{n}$ and $\Lambda_{g}^{n}$ may be discarded when no longer needed.

The predominant challenge in the discretization and solution of Eqs. (21)-(23) is the infrastructure required to simultaneously manage data from several time levels. Inspection of Eqns. (42)-(44) in the appendix that are higher-order analogs to Eq. (21) shows that for a given time step $n$, the solution for $\boldsymbol{\Lambda}_{g}^{n}$ may depend on values of $\mathbf{Q}$ from adjacent time levels both prior and subsequent to level $n$. Values of $\boldsymbol{\Lambda}_{f}$ must also be available at time level $n$ as well as later time levels. Moreover, this complexity increases with the temporal order of the scheme.

The summation term in Eq. (21) is ultimately due to the dependency of the mesh speeds on grid coordinates at multiple time levels, according to the BDF scheme being used. Rather than linearizing $\mathbf{R}$

and $\mathcal{R}_{G C L}$ at several time levels with respect to the grid coordinates at the current time level as indicated in the summation, an inverse approach more amenable to the existing implementation of the spatial linearizations is used. The residual at time level $n$ is linearized with respect to the grid coordinates at every time level in the temporal stencil by seeding the linearizations with the appropriate BDF coefficient. The results are then stored temporarily for use in evaluating the summation term at subsequent time levels within the stencil, after which the linearizations are discarded. 


\section{Verification of Adjoint Implementation}

To verify the accuracy of the implementation, comparisons are made with results generated through an independent approach based on the use of complex variables. This approach was originally suggested in Refs. [31] and [32], and was first applied to a Navier-Stokes solver in Ref. [3]. Using this formulation, an expression for the derivative of a real-valued function $f(x)$ may be found by expanding the function in a complex-valued Taylor series, using an imaginary perturbation $i \varepsilon$ :

$$
\frac{\partial f}{\partial x}=\frac{\operatorname{Im}[f(x+i \varepsilon)]}{\varepsilon}+O\left(\varepsilon^{2}\right) .
$$

The primary advantage of this method is that true second-order accuracy may be obtained by selecting step sizes without concern for subtractive cancellation error typically present in real-valued divided differences. Through the use of an automated scripting procedure outlined in Ref. [33], this capability can be immediately recovered at any time for the baseline flow solver. For computations using this method, the imaginary step size has been chosen to be $10^{-30}$. For each verification test, all equations sets are converged to machine precision for both the complex-variable and adjoint approaches. When used, the elasticity matrix $\mathcal{K}$ is assumed to be constant throughout the verification.

\section{Static Grid}

\section{Test Case}

The first test case is used to verify the implementation for unsteady flows on static grids. For this example, fully turbulent flow over the ONERA M6 wing ${ }^{[34]}$ shown in Fig. 1 is considered. The grid contains 16,391 nodes and 90,892 tetrahedral elements and 16 processors are used for the simulation. The freestream Mach number is 0.3 , the angle of attack is $1^{\circ}$, and the Reynolds number is 1 million based on the mean aerodynamic chord (MAC). The simulation is initiated from freestream conditions $\mathbf{Q}^{\infty}$, which leads to $\mathbf{R}^{i n} \equiv \mathbf{Q}^{\infty}-\mathbf{Q}^{0}$. The solution is advanced 5 physical time steps using a nondimensional $\Delta t$ of 0.1 . While this coarse spatial resolution, relatively large time step, and brief duration of the simulation are not sufficient to resolve the flow physics of the problem, they are adequate to evaluate the discrete consistency of the implementation.

\section{Design Variables}

For this test, two general classes of design variables are used. The first class of variables is comprised of global parameters unrelated to the computational grid. These variables include parameters such as the freestream Mach number and angle of attack. Such variables are useful in verifying the implementation of the flowfield adjoint equation, as the terms in Eq. (23) associated with these parameters are generally trivial to implement or identically zero, and solution of the mesh adjoint equations is not required.

The second class of design variables provides general shape control of the configuration. The implementation allows the user to employ a geometric parameterization scheme of choice, provided the associated surface grid linearizations are available. For all examples in the current study, the grid parameterization approach described in Ref. [35] is used. This approach can be used to define general shape parameterizations of existing grids using a set of aircraft-centric design variables such as camber, thickness, shear, twist, and planform parameters at various locations on the geometry. The user also has the freedom to associate two or more design variables to define more general parameters. In the event that multiple bodies of the same shape are to be designed, the implementation allows for a single set of design variables to be used to simultaneously define such bodies. In this fashion, the shape of each body is constrained to be identical throughout the course of the design.

\section{Grid Adjoint Equation}

For this case, there is only one grid operator, $\mathbf{G}(\mathbf{X}, \mathbf{D}) \equiv \mathbf{X}_{\text {surf }}-\mathcal{K} \mathbf{X}$, which does not depend on time. As a result, the grid adjoint equation can be recast as

$$
\begin{gathered}
{\left[\mathcal{K}+\frac{\partial \mathcal{K}}{\partial \mathbf{X}} \mathbf{X}\right]^{T} \boldsymbol{\Lambda}_{g}=\sum_{n=1}^{N}\left\{\left[\frac{\partial f^{n}}{\partial \mathbf{X}}\right]^{T}+\left[\frac{\partial \mathcal{V}^{n}}{\partial \mathbf{X}} \frac{\mathbf{Q}^{n}-\mathbf{Q}^{n-1}}{\Delta t}\right]^{T} \boldsymbol{\Lambda}_{f}^{n}+\sum_{k=0}^{1}\left[\frac{\partial \mathbf{R}^{n+k}}{\partial \mathbf{X}}\right]^{T} \boldsymbol{\Lambda}_{f}^{n+k}\right\}} \\
+\left[\frac{\partial f^{0}}{\partial \mathbf{X}}\right]^{T}+\left[\frac{\partial \mathbf{R}^{1}}{\partial \mathbf{X}}\right]^{T} \mathbf{\Lambda}_{f}^{1}
\end{gathered}
$$


and the sensitivity derivative is

$$
\frac{d L}{d \mathbf{D}}=\sum_{n=1}^{N}\left(\frac{\partial f^{n}}{\partial \mathbf{D}}+\left[\boldsymbol{\Lambda}_{f}^{n}\right]^{T} \frac{\partial \mathbf{R}^{n}}{\partial \mathbf{D}}\right) \Delta t+\left(\frac{\partial f^{0}}{\partial \mathbf{D}}+\left[\boldsymbol{\Lambda}_{f}^{0}\right]^{T} \frac{\partial \mathbf{Q}^{\infty}}{\partial \mathbf{D}}\right) \Delta t+\left[\boldsymbol{\Lambda}_{g}\right]^{T}\left[\frac{\partial \mathbf{X}_{\text {surf }}}{\partial \mathbf{D}}-\frac{\partial \mathcal{K}}{\partial \mathbf{D}} \mathbf{X}\right] \Delta t .
$$

\section{Computational Results}

The test has been performed using the BDF1 scheme and all other time-integration schemes described in the appendix, and results are listed in Table 1. Sensitivity derivatives of the lift coefficient at the final time step with respect to the angle of attack and a camber variable located at the midspan of the wing are shown. The results for the adjoint implementation exhibit excellent agreement with the complex-variable approach, differing at most in the fifteenth digit.

\section{Rigidly-Moving Grid}

\section{Test Case}

The next test case is used to verify the implementation for rigidly moving meshes. For this case, the grid and freestream conditions and computational environment are identical to those described for the previous test; however, the mesh is now subjected to an oscillatory pitch-plunge motion based on the rigid mesh transform approach outlined earlier. The nondimensional pitching and plunging reduced frequencies are 0.5 and 0.1 , respectively. The pitching amplitude is $5^{\circ}$ and takes place about a vector normal to the symmetry plane located $0.47 \mathrm{MAC}$ from the wing root leading edge. The amplitude of the plunging motion is $0.38 \mathrm{MAC}$. The baseline wing position at $t=0$ is as shown in Fig. 1. As in the prior test, the simulation is initiated from freestream conditions $\mathbf{R}^{i n} \equiv \mathbf{Q}^{\infty}-\mathbf{Q}^{0}$ and is advanced 5 physical time steps using a nondimensional $\Delta t$ of 0.1 .

\section{Design Variables}

The design variables for the current test include those described above for the static grid example, as well as a third class of parameters governing the rigid motion procedure described above. These include translation and rotation frequencies, amplitudes, and directional vectors, as well as centers of rotation.

\section{Grid Adjoint Equation}

For this test case, the following grid operators are used: at the zeroth time level, the grid is either unchanged or governed by the elasticity equations $\mathbf{G}^{0}\left(\mathbf{X}^{0}, \mathbf{D}\right) \equiv \mathbf{X}_{\text {surf }}^{0}-\mathcal{K}^{0} \mathbf{X}^{0}$; grids at other time levels are governed by the rigid motion equation $\mathbf{G}^{n}\left(\mathbf{X}^{n}, \mathbf{D}\right) \equiv \mathcal{R}^{n} \mathbf{X}^{0}+\tau^{n}-\mathbf{X}^{n}$.

The grid adjoint equations are given by

$$
\boldsymbol{\Lambda}_{g}^{n}=\left[\frac{\partial f}{\partial \mathbf{X}^{n}}\right]^{T}+\left[\frac{\partial \mathcal{V}^{n}}{\partial \mathbf{X}^{n}} \frac{\mathbf{Q}^{n}-\mathbf{Q}^{n-1}}{\Delta t}\right]^{T} \boldsymbol{\Lambda}_{f}^{n}+\sum_{k=0}^{1}\left[\frac{\partial \mathbf{R}^{n+k}}{\partial \mathbf{X}^{n}}+\frac{\partial \mathcal{R}_{G C L}^{n+k}}{\partial \mathbf{X}^{n}} \mathbf{Q}^{n+k-1}\right]^{T} \boldsymbol{\Lambda}_{f}^{n+k}, \text { for } 1 \leq n \leq N
$$

Under the assumption that the shape does not change ( $\mathbf{X}^{0}$ is constant), the sensitivity derivative is given by

$$
\begin{aligned}
\frac{d L}{d \mathbf{D}}=\sum_{n=1}^{N}\left(\frac{\partial f^{n}}{\partial \mathbf{D}}+\left[\boldsymbol{\Lambda}_{f}^{n}\right]^{T}\right. & {\left.\left[\frac{\partial \mathbf{R}^{n}}{\partial \mathbf{D}}+\frac{\partial \mathcal{R}_{G C L}^{n}}{\partial \mathbf{D}} \mathbf{Q}^{n-1}\right]+\left[\boldsymbol{\Lambda}_{g}^{n}\right]^{T} \frac{\partial\left(\mathcal{R}^{n} \mathbf{X}^{0}+\tau^{n}\right)}{\partial \mathbf{D}}\right) \Delta t } \\
& +\left(\frac{\partial f^{0}}{\partial \mathbf{D}}+\left[\boldsymbol{\Lambda}_{f}^{0}\right]^{T} \frac{\partial \mathbf{Q}^{\infty}}{\partial \mathbf{D}}\right) \Delta t .
\end{aligned}
$$

The formulation that would allow shape design is the following:

$$
\left[\mathcal{K}^{0}+\frac{\partial \mathcal{K}^{0}}{\partial \mathbf{X}^{0}} \mathbf{X}^{0}\right]^{\mathrm{T}} \boldsymbol{\Lambda}_{g}^{0}=\left(\sum_{n=1}^{N}\left[\mathcal{R}^{n}\right]^{T} \boldsymbol{\Lambda}_{g}^{n}\right)+\left[\frac{\partial f^{0}}{\partial \mathbf{X}^{0}}\right]^{T}+\left[\frac{\partial \mathbf{Q}^{\infty}}{\partial \mathbf{X}^{0}}\right]^{T} \boldsymbol{\Lambda}_{f}^{0}+\left[\frac{\partial \mathbf{R}^{1}}{\partial \mathbf{X}^{0}}+\frac{\partial \mathcal{R}_{G C L}^{1}}{\partial \mathbf{X}^{0}} \mathbf{Q}^{\infty}\right]^{T} \boldsymbol{\Lambda}_{f}^{1}
$$

and the corresponding sensitivity derivative is

$$
\frac{d L}{d \mathbf{D}}=\sum_{n=1}^{N}\left(\frac{\partial f^{n}}{\partial \mathbf{D}}+\left[\boldsymbol{\Lambda}_{f}^{n}\right]^{T}\left[\frac{\partial \mathbf{R}^{n}}{\partial \mathbf{D}}+\frac{\partial \mathcal{R}_{G C L}^{n}}{\partial \mathbf{D}} \mathbf{Q}^{n-1}\right]+\left[\boldsymbol{\Lambda}_{g}^{n}\right]^{T} \frac{\partial \tau^{n}}{\partial \mathbf{D}}\right) \Delta t
$$




$$
+\left(\frac{\partial f^{0}}{\partial \mathbf{D}}+\left[\boldsymbol{\Lambda}_{f}^{0}\right]^{T} \frac{\partial \mathbf{Q}^{\infty}}{\partial \mathbf{D}}+\left[\boldsymbol{\Lambda}_{g}^{0}\right]^{T}\left[\frac{\partial \mathbf{X}_{\text {surf }}^{0}}{\partial \mathbf{D}}-\frac{\partial \mathcal{K}^{0}}{\partial \mathbf{D}} \mathbf{X}^{0}\right]\right) \Delta t .
$$

\section{Computational Results}

Results for the derivatives of the lift coefficient at the final time step are shown in Table 2 for the current case. In addition to the angle of attack and camber variables, derivatives with respect to the rigid motion pitching frequency are also shown. The agreement with the complex-variable formulation is excellent for each of the time integration schemes considered.

\section{Morphing Grid}

\section{Test Case}

To evaluate the accuracy of the implementation for morphing grids, the test case used for rigid motion described above is repeated with slight modifications. For the current test, the surface grid of the wing is moved using rigid motion, while the interior of the mesh is determined using the elasticity relation given by Eq. (9). All other input parameters remain unchanged.

\section{Design Variables}

The current test case uses the same design variables as the rigid motion test case described above.

\section{Grid Adjoint Equation}

At all time levels, the grids are governed by the elasticity equations $\mathbf{G}^{n}\left(\mathbf{X}^{n}, \mathbf{D}\right) \equiv \mathbf{X}_{\text {surf }}^{n}-\mathcal{K}^{0} \mathbf{X}^{n}$, and the surface coordinates are governed by the rigid motion equation $\mathbf{X}_{\text {surf }}^{n}=\mathcal{R}^{n} \mathbf{X}_{\text {surf }}^{0}+\tau^{n}$.

The grid adjoint equations are given by

$$
\begin{gathered}
{\left[\mathcal{K}^{0}\right]^{T} \boldsymbol{\Lambda}_{g}^{n}=\left[\frac{\partial f}{\partial \mathbf{X}^{n}}\right]^{T}+\left[\frac{\partial \mathcal{V}^{n}}{\partial \mathbf{X}^{n}} \frac{\mathbf{Q}^{n}-\mathbf{Q}^{n-1}}{\Delta t}\right]^{T} \boldsymbol{\Lambda}_{f}^{n}+\sum_{k=0}^{1}\left[\frac{\partial \mathbf{R}^{n+k}}{\partial \mathbf{X}^{n}}+\frac{\partial \mathcal{R}_{G C L}^{n+k}}{\partial \mathbf{X}^{n}} \mathbf{Q}^{n+k-1}\right]^{T} \boldsymbol{\Lambda}_{f}^{n+k},} \\
{\left[\mathcal{K}^{0}+\frac{\partial \mathcal{K}^{0}}{\partial \mathbf{X}^{0}} \mathbf{X}^{0}\right]^{T} \boldsymbol{\Lambda}_{g}^{0}=\left[\frac{\partial f^{0}}{\partial \mathbf{X}^{0}}\right]^{T}+\left[\frac{\partial \mathbf{Q}^{\infty}}{\partial \mathbf{X}^{0}}\right]^{T} \boldsymbol{\Lambda}_{f}^{0}+\left[\frac{\partial \mathbf{R}^{1}}{\partial \mathbf{X}^{0}}+\frac{\partial \mathcal{R}_{G C L}^{1}}{\partial \mathbf{X}^{0}} \mathbf{Q}^{\infty}\right]^{T} \boldsymbol{\Lambda}_{f}^{1}-\sum_{n=1}^{N}\left[\frac{\partial \mathcal{K}^{0}}{\partial \mathbf{X}^{0}} \mathbf{X}^{n}\right]^{T} \boldsymbol{\Lambda}_{g}^{n} .}
\end{gathered}
$$

The sensitivity derivative is

$$
\begin{aligned}
\frac{d L}{d \mathbf{D}}=\sum_{n=1}^{N}\left(\frac{\partial f^{n}}{\partial \mathbf{D}}+\right. & {\left.\left[\boldsymbol{\Lambda}_{f}^{n}\right]^{T}\left[\frac{\partial \mathbf{R}^{n}}{\partial \mathbf{D}}+\frac{\partial \mathcal{R}_{G C L}^{n}}{\partial \mathbf{D}} \mathbf{Q}^{n-1}\right]+\left[\boldsymbol{\Lambda}_{g}^{n}\right]^{T}\left[\frac{\partial\left(\mathcal{R}^{n} \mathbf{X}_{\text {surf }}^{0}+\tau^{n}\right)}{\partial \mathbf{D}}-\frac{\partial \mathcal{K}^{0}}{\partial \mathbf{D}} \mathbf{X}^{n}\right]\right) \Delta t } \\
& +\left(\frac{\partial f^{0}}{\partial \mathbf{D}}+\left[\boldsymbol{\Lambda}_{f}^{0}\right]^{T} \frac{\partial \mathbf{Q}^{\infty}}{\partial \mathbf{D}}+\left[\boldsymbol{\Lambda}_{g}^{0}\right]^{T}\left[\frac{\partial \mathbf{X}_{\text {surf }}^{0}}{\partial \mathbf{D}}-\frac{\partial \mathcal{K}^{0}}{\partial \mathbf{D}} \mathbf{X}^{0}\right]\right) \Delta t .
\end{aligned}
$$

Two observations can be made. First, note that in the absence of any surface motion, i.e., $\mathcal{R}^{n}$ is the identity matrix and $\tau^{n}=0$, the morphing grid formulation is equivalent to the static grid formulation. Also, with a constant transformation matrix $\mathcal{T}$ applied to all computational boundaries, the morphing and rigidly moving grid formulations are equivalent.

\section{Computational Results}

The results for the current test case are shown in Table 3. Derivatives of the lift coefficient at the final time step with respect to each of the design variables exhibit excellent agreement for the adjoint implementation and complex variable formulation.

\section{Large-Scale Design Cases}

Two large-scale design optimization examples are presented. Although the grid motion in both cases is prescribed, a more realistic treatment would involve the use of additional coupled computational models such as six degree-of-freedom or structural simulations. While such capabilities are available for use with 
the flow solver, ${ }^{[19]}$ their effects have not been accounted for in the derivation and implementation of the adjoint equations. This important development is relegated to future work.

Both of the example cases shown below have been performed using 128 dual-socket quad-core nodes with $3.0 \mathrm{GHz}$ Intel Xeon processors in a fully-dense fashion for a total of 1,024 computational cores. This environment has been chosen to maximize computational efficiency for the chosen test problems; numerical experiments have shown that the solvers used in the current study scale well in this range for the grid sizes selected.

The computational grid sizes and time steps for the examples presented here have been chosen merely to demonstrate optimization capability for typical problems using immediately available resources. Spatial and/or temporal refinement could be readily performed if desired. Although the formulation places no restrictions on initial conditions, all solutions are started from freestream conditions. The grids have been generated using the method of Ref. [36] and the optimizations have been performed using the package described in Ref. [37].

\section{Tiltrotor Configuration}

The first large-scale example is a three-bladed tiltrotor configuration similar to that used by the V-22 aircraft and is based on the Tilt Rotor Aeroacoustics Model (TRAM) geometry described in Refs. [38] and [39]. The grid used for this computation is designed for a blade collective setting of $\Theta=14^{\circ}$ and consists of 5,048,727 nodes and 29,802,252 tetrahedral elements. The rotational speed of the rotor is held constant at a value corresponding to a tip Mach number of 0.62 in a hover condition. The Reynolds number is 2.1 million based on the blade tip chord. The physical time step is chosen to correspond to $1^{\circ}$ of rotor azimuth, for a total of 360 time steps per revolution. The $\mathrm{BDF} 2_{\mathrm{opt}}$ formulation outlined in Ref. [40] is used with 10 subiterations per time step.

For this test, the prescribed rigid mesh motion consists of 4 initial revolutions of the geometry designed to reach a quasi-steady hover condition, followed by five additional revolutions during which a $90^{\circ}$ constant-rate pitch-up maneuver into a forward-flight mode is performed. A more realistic pitch-up scenario might consist of many more revolutions; however, the prescribed motion was chosen to keep the cost of the computation affordable on the current resources. During the pitch-up phase of the motion, an assumed forward-flight velocity profile based on a simple sine function is imposed through the mesh speed terms. The schedule for the shaft angle and forward-flight velocity is shown in Fig. 2, where the shaft angle is defined to be $0^{\circ}$ in the hover condition and $90^{\circ}$ in forward flight. The resulting motion is shown in Fig. 3 , where a snapshot of the rotor is shown every $360^{\circ}$ during the course of the motion. An isosurface of the second invariant of the velocity-gradient tensor, also known as the Q criterion from Ref. [41], at the time step corresponding to $\Psi=1440^{\circ}$ is shown in Fig. 4. The tip vortex system is maintained for 2 to 3 revolutions of the rotor.

The objective function for the current test case is to maximize the rotor thrust coefficient over the time interval corresponding to the pitch-up maneuver, $1441^{\circ} \leq \Psi \leq 3240^{\circ}$ :

$$
f=\sum_{n=1441}^{3240}\left(C_{T}^{n}-0.1\right)^{2} \Delta t .
$$

Here, the target thrust coefficient value of 0.1 has been chosen to sufficiently exceed the baseline thrust profile shown as the solid line in Fig. 5. After the first four rotor revolutions, the thrust coefficient has reached a quasi-steady value of approximately 0.015 , which is in good agreement with experimental data given in Refs. [38] and [39]. The thrust coefficient shows a discontinuous behavior at the impulsive start of the pitch-up motion $(n=1441)$ and gradually decreases to a lower constant value in the forward-flight condition. A subtle 3/rev oscillation in the thrust coefficient during the pitch-up maneuver can also be seen.

The surface grid has been parameterized as described in Ref. [42]. This approach yields a set of 44 active design variables describing the thickness and camber of the blade geometry as shown in Fig. 6; thinning of the blade is not allowed. In addition, a single twist variable is used to modify the blade collective setting during the design.

The convergence history for six design cycles is shown in Fig. 7. The optimizer quickly reduces the value of the objective function over the first two design cycles, after which further improvements are minimal. Closer inspection of the design variables indicates that the majority of values have reached their bound constraints, preventing any further reduction in the objective function. The final thrust coefficient 
profile is included as the dashed line in Fig. 5. Cross-sections of the baseline blade geometry are compared with the optimized geometry in Fig. 8. The optimization has increased the camber of the blade across the span, as well as the blade collective setting.

The cost of each solution to the unsteady flow and adjoint equations for the current example is approximately 3.5 and 10.5 wallclock hours, respectively; however, due to frequent file $\mathrm{I} / \mathrm{O}$, this estimate varies with file system load. The optimization procedure requires twelve calls to the flow solver and six calls to the adjoint solver, for a total runtime of approximately 4.5 days of wallclock time or 110,000 CPU hours. The disk storage required for one complete flow solution is approximately 1.5 terabytes.

\section{Fighter Jet with Simulated Aeroelastic Effects}

The second example uses a deforming grid approach to simulate aeroelastic motion of the modified F15 fighter jet configuration described in Ref. [43] and known as NASA research aircraft \#837, shown in Fig. 9. The computational model assumes half-plane symmetry in the spanwise direction. The grid consists of 4,715,852 nodes and 27,344,343 tetrahedral elements and includes detailed features of the external airframe as well as the internal ducting upstream of the engine fan face and plenum/nozzle combination downstream of the turbine. For the current test, the freestream Mach number is 0.90 , the angle of attack is $0^{\circ}$, and the Reynolds number based on the MAC is 1 million. The static pressure ratio at the engine fan face is set to 0.9 and the total pressure ratio at the plenum face is ramped linearly from 1.0 to its final value of 5.0 over the first 50 time steps.

The prescribed grid motion consists of $5 \mathrm{~Hz} 0.3^{\circ}$ oscillatory rotations of the canard, wing, and tail surfaces about their root chordlines, with the wing oscillations $180^{\circ}$ out of phase with the canard and tail motion. In addition, the main wing is also subjected to a $5 \mathrm{~Hz}$ oscillatory twisting motion whose amplitude decays linearly from $0.5^{\circ}$ at the wing tip to $0^{\circ}$ at the wing root and takes place about the quarter-chord line. This composite motion results in a maximum wing tip deflection of approximately $1.3 \%$ MAC as shown in Fig. 10. The BDF $2_{\text {opt }}$ scheme is used with 10 subiterations and a physical time step corresponding to 100 steps per cycle of grid motion.

The unsteady lift-to-drag ratio $(L / D)$ for the baseline configuration undergoing the specified motion for 300 time steps is shown as the solid line in Fig. 11. The $L / D$ behavior begins to exhibit a periodic response after approximately 100 time steps. The high-frequency oscillations in the profile are believed to be due to a small unsteadiness in the engine plume shown in Fig. 12; this behavior is also present when the mesh is held fixed.

The objective function for the current test case is to maximize $L / D$ on the interval $201 \leq n \leq 300$ :

$$
f=\sum_{n=201}^{300}\left[(L / D)^{n}-5.0\right]^{2} \Delta t,
$$

where the target $L / D$ value of 5.0 has been chosen to provide sufficient room for optimization over the baseline profile. The surface grids for the canard, wing, and tail have been parameterized as shown in Fig. 13, resulting in a set of 98 active design variables describing the thickness and camber of each surface. Thinning of the geometry is not permitted.

Convergence of the objective function is shown in Fig. 14. A large reduction in the function is obtained after a single design cycle, after which further improvements are minimal due to many of the design variables having reached their bound constraints. The final $L / D$ profile is included as the dashed line in Fig. 11. The resulting shape changes at various spanwise stations on the canard, wing, and tail are shown in Fig. 15, where the vertical scale has been exaggerated for clarity. The design procedure has increased the thickness of the wing and canard, as well as the camber across all three elements. Closer inspection shows that the trailing edges of each surface have also been deflected in a downward fashion.

The wallclock times required for single flow and adjoint solutions for the current problem are approximately 1 and 1.5 hours, respectively. For the five design cycles shown in Fig. 14, the optimizer requires ten flow solutions and five adjoint solutions, or a total wallclock time of approximately 18 hours or 18,400 CPU hours. The disk space necessary to store a single unsteady flow solution is 136 gigabytes. 


\section{Summary and Future Work}

A discrete adjoint-based methodology for optimization of unsteady flows governed by the threedimensional Reynolds-averaged Navier-Stokes equations on dynamic unstructured grids has been formulated and implemented. The methodology accounts for mesh motion based on both rigid movement as well as deforming grids. The accuracy of the implementation has been verified using comparisons with an independent approach based on the use of complex variables. The methodology has been successfully used in a massively parallel environment to perform two large-scale design optimization examples: one for a tiltrotor in a pitch-up maneuver into a forward-flight regime and another for a fighter jet with simulated aeroelastic effects.

Although the approach outlined in the current study represents significant progress towards the goal of performing routine optimization of unsteady turbulent flows, a number of research areas remain to be explored. The extension of the present formulation to overset grid topologies is ongoing and will allow the treatment of multiple bodies undergoing large relative motion. Methods aimed at reducing the storage costs associated with the flow solution have the potential to drastically reduce disk requirements. Techniques based on variable or adaptive time steps as well as alternate time integration schemes should be examined. The effects of related computational disciplines such as six degree-of-freedom and structural models should also be properly accounted for. Finally, the use of the unsteady flowfield adjoint solution holds tremendous potential for performing mathematically rigorous mesh adaptation to specified error bounds.

\section{Acknowledgments}

The authors wish to thank Dr. Robert Biedron of NASA Langley Research Center for many useful discussions pertaining to the current work. Geometric parameterizations provided by Bill Jones of NASA Langley Research Center are also appreciated. Jan-Renee Carlson of NASA Langley Research Center is acknowledged for her assistance with plenum geometry modifications and boundary conditions for the fighter jet example. The second and third authors acknowledge the support from NASA under grant NNL07AA23C.

\section{References}

${ }^{[1]}$ Vanderplaats, G.N., Hicks, R.N., and Murman, E.M., "Application of Numerical Optimization Techniques to Airfoil Design," NASA Conference on Aerodynamic Analysis Requiring Advanced Computers," NASA SP-347, Part II, March 1975.

${ }^{[2]}$ Baysal, O., and Eleshaky, M.E., "Aerodynamic Sensitivity Analysis Methods for the Compressible Euler Equations," Journal of Fluids Engineering, Vol. 113, 1991, pp. 681-688.

${ }^{[3]}$ Anderson, W.K., Newman, J.C., Whitfield, D.L., and Nielsen, E.J., "Sensitivity Analysis for the NavierStokes Equations on Unstructured Meshes Using Complex Variables," AIAA Journal, Vol. 39, No. 1, 2001, pp. 56-63.

${ }^{[4]}$ Nielsen, E.J. and Park, M.A., "Using An Adjoint Approach to Eliminate Mesh Sensitivities in Computational Design," AIAA Journal, Vol. 44, No. 5, 2006, pp. 948-953.

${ }^{[5]}$ Newman III, J.C., Taylor III, A.C., Barnwell, R.W., Newman, P.A., and Hou, G.J.-W., "Overview of Sensitivity Analysis and Shape Optimization for Complex Aerodynamic Configurations," Journal of Aircraft, Vol. 36, No. 1, 1999, pp. 87-96.

${ }^{[6]}$ Fidkowski, K.J., and Darmofal, D.L., "Output-Based Error Estimation and Mesh Adaptation in Computational Fluid Dynamics: Overview and Recent Results," AIAA 2009-1303, 2009.

${ }^{[7]}$ Nadarajah, S. and Jameson, A., "Optimal Control of Unsteady Flows Using Time Accurate and NonLinear Frequency Domain Methods," AIAA 2002-5436, 2002.

${ }^{[8]}$ Choi, S., Potsdam, M., Lee, K., Iaccarino, G., and Alonso, J.J., "Helicopter Rotor Design Using a TimeSpectral and Adjoint-Based Method," AIAA 2008-5810, 2008.

${ }^{[9]}$ Gunzburger, M.D., Perspectives in Flow Control and Optimization, SIAM, Philadelphia, 2003.

${ }^{[10]}$ Mavriplis, D.J., "Solution of the Unsteady Discrete Adjoint for Three-Dimensional Problems on Dynamically Deforming Unstructured Meshes," AIAA 2008-727, 2008.

${ }^{[11]}$ Muldoon, F., "Control of a Simplified Unsteady Film-Cooling Flow Using Gradient-Based Optimization," AIAA Journal, Vol. 46, No. 10, 2008, pp. 2443-2458.

${ }^{[12]}$ Rumpfkeil, M.P., and Zingg, D.W., "A General Framework for the Optimal Control of Unsteady Flows with Applications," AIAA 2007-1128, 2007. 
${ }^{[13]}$ Yamaleev, N.K., Diskin, B., and Nielsen, E.J., "Adjoint-Based Methodology for Time-Dependent Optimization," AIAA 2008-5857, 2008.

${ }^{[14]}$ Nielsen, E.J., "Aerodynamic Design Sensitivities on an Unstructured Mesh Using the Navier-Stokes Equations and a Discrete Adjoint Formulation," Ph.D. Dissertation, Dept. of Aerospace and Ocean Engineering, Virginia Polytechnic Inst. and State Univ., December 1998.

${ }^{[15]}$ Nielsen, E.J. and Anderson, W.K., "Aerodynamic Design Optimization on Unstructured Meshes Using the Navier-Stokes Equations," AIAA Journal, Vol. 37, No. 11, 1999, pp. 1411-1419.

${ }^{[16]}$ Nielsen, E.J. and Anderson, W.K., "Recent Improvements in Aerodynamic Design Optimization on Unstructured Meshes," AIAA Journal, Vol. 40, No. 6, 2002, pp. 1155-1163.

${ }^{[17]}$ Nielsen, E.J., Lu, J., Park, M.A., and Darmofal, D.L., "An Implicit, Exact Dual Adjoint Solution Method for Turbulent Flows on Unstructured Grids," Computers and Fluids, Vol. 33, No. 9, 2004, pp. 1131-1155.

${ }^{[18]}$ Nielsen, E.J. and Kleb, W.L., "Efficient Construction of Discrete Adjoint Operators on Unstructured Grids by Using Complex Variables," AIAA Journal, Vol. 44, No. 4, 2006, pp. 827-836.

${ }^{[19]}$ Biedron, R.T. and Thomas, J.L., "Recent Enhancements to the FUN3D Flow Solver for Moving Mesh Applications," AIAA 2009-1360, 2009.

${ }^{[20]}$ Thomas, P.D. and Lombard, C.K., "Geometrical Conservation Law and Its Application to Flow Computations on Moving Grids," AIAA Journal, Vol. 17, No. 10, 1978, pp. 1030-1037.

${ }^{[21]}$ Anderson, W. K. and Bonhaus, D.L., "An Implicit Upwind Algorithm for Computing Turbulent Flows on Unstructured Grids," Computers and Fluids, Vol. 23, No. 1, 1994, pp. 1-21.

${ }^{[22]}$ http://fun3d.larc.nasa.gov

${ }^{[23]}$ Roe, P.L., “Approximate Riemann Solvers, Parameter Vectors, and Difference Schemes,” Journal of Computational Physics, Vol. 43, No. 2, 1981, pp. 357-372.

${ }^{[24]}$ Spalart, P.R. and Allmaras, S.R., "A One-Equation Turbulence Model for Aerodynamic Flows," AIAA 92-0439, 1991.

${ }^{[25]}$ Saad, Y., and Schultz, M.H., "GMRES: A Generalized Minimal Residual Algorithm for Solving Nonsymmetric Linear Systems," SIAM Journal of Scientific and Statistical Computing, Vol. 7, No. 3, 1986, pp. 856-869.

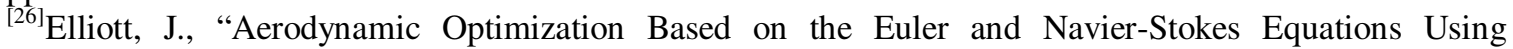
Unstructured Grids," Ph.D. Dissertation, Dept. of Aeronautics and Astronautics, Massachusetts Inst. Of Technology, Cambridge, MA, June 1998.

${ }^{[27]}$ Campobasso, M., and Giles, M., "Effects of Flow Instabilities on the Linear Analysis of Turbomachinery Aeroelasticity," Journal of Propulsion and Power, Vol. 19, No. 2, 2003, pp. $250-259$.

${ }^{[28]}$ Eisenstat, S.C., Elman, H.C., and Schultz, M., "Variational Iterative Methods for Nonsymmetric Systems of Linear Equations," SIAM Journal of Numerical Analysis, Vol. 20, No. 2, 1983, pp. 345-357.

${ }^{\left[{ }^{29]} H i n z e, ~ M ., ~ W a l t h e r, ~ A ., ~ a n d ~ S t e r n b e r g, ~ J ., ~ " D i s c r e t e ~ A p p r o x i m a t i o n ~ S c h e m e s ~ f o r ~ R e d u c e d ~ G r a d i e n t s ~ a n d ~\right.}$ Reduced Hessians in Navier-Stokes Control Utilizing an Optimal Memory-Reduced Procedure for Calculating Adjoints," Optimal Control Applications and Methods, Vol. 27, No. 1, 2006, pp. 19-40.

${ }^{[30]}$ Yamaleev, N., Diskin, B., and Nielsen, E., "Locally Optimal Control/Design Strategies for Unsteady Compressible Flows," AIAA 2009-1169, January 2009.

${ }^{[31]}$ Lyness, J.N., "Numerical Algorithms Based on the Theory of Complex Variables," Proc. ACM 22nd Nat. Conf., Thomas Book Co., Washington, D.C., 1967, pp. 124-134.

${ }^{[32]}$ Lyness, J.N. and Moler, C.B., "Numerical Differentiation of Analytic Functions," SIAM Journal of Numerical Analysis, Vol. 4, 1967, pp. 202-210.

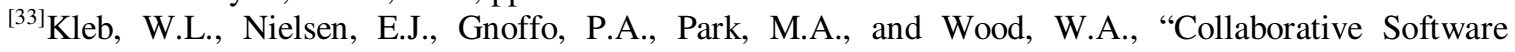
Development in Support of Fast Adaptive Aerospace Tools (FAAST),” AIAA 2003-3978, 2003.

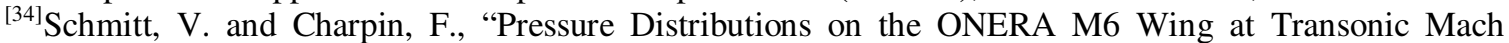
Numbers," Experimental Database for Computer Program Assessment, AGARD-AR-138, May 1979, pp. B1-1-B1-44.

${ }^{[35]}$ Samareh, J.A., “A Novel Shape Parameterization Approach,” NASA TM-1999-209116, May 1999.

${ }^{[36]}$ Pirzadeh, S., "Three-Dimensional Unstructured Viscous Grids by the Advancing Front Method," AIAA Journal, Vol. 34, No. 1, 1996, pp. 43-49.

${ }^{[37]}$ Kaufman, L. and Gay, D., "PORT Library: Optimization and Mathematical Programming - User's Manual," Bell Laboratories, 1997.

${ }^{[38]}$ Young, L.A., Booth, Jr., E.R., Yamauchi, G.K., Botha, G., and Dawson, S., "Overview of the Testing of a Small-Scale Proprotor," American Helicopter Society 55 ${ }^{\text {th }}$ Annual Forum, Montreal, Canada, May 1999. 
${ }^{[39]}$ Swanson, S.M., McCluer, M.S., Yamauchi, G.K., and Swanson, A.A., "Airloads Measurements from a 1/4-Scale Tiltrotor Wind Tunnel Test," $25^{\text {th }}$ European Rotorcraft Forum, Rome, Italy, September 1999.

${ }^{[40]}$ Nyukhtikov, M., Smelova, N., Mitchell, B.E., and Holmes, D.G., "Optimized Dual-Time Stepping Technique for Time-Accurate Navier-Stokes Calculation," Proceedings of the $10^{\text {th }}$ International Symposium on Unsteady Aerodynamics, Aeroacoustics, and Aeroelasticity of Turbomachines, 2003.

${ }^{[41]} J$ eong, J., and Hussain, F., "On the Identification of a Vortex," Journal of Fluid Mechanics," Vol. 285, 1985, pp. 69-94.

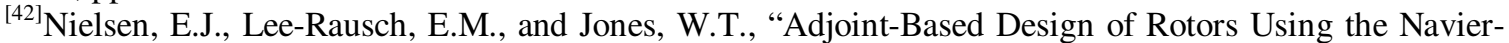
Stokes Equations in a Noninertial Reference Frame," American Helicopter Society $65^{\text {th }}$ Annual Forum, Grapevine, TX, May 2009.

${ }^{[43]}$ http://www.nasa.gov/centers/dryden/aircraft/F-15B-837/index.html

\section{Appendix: Adjoint Equations for Higher Order BDF Schemes}

The high (up to third) order BDF discretizations for the time derivative of a function $s$ are defined as

$$
\frac{\partial s}{\partial t}=\frac{1}{\Delta t}\left[a s^{n}+b s^{n-1}+c s^{n-2}+d s^{n-3}\right]
$$

where $n$ is a time level, and the coefficients are given in Table 4 . The coefficients listed for the BDF $2_{\text {opt }}$ scheme are a linear combination of the BDF2 and BDF3 coefficients taken from Ref. [40]. The resulting scheme is second-order accurate but has a leading truncation error term less than that of the BDF2 scheme. While usually found to be stable in practice, stability of the BDF2 $2_{\text {opt }}$ and third-order BDF3 scheme are not guaranteed. Discrete conservation laws are defined as

$$
a \mathcal{V}^{n} \frac{\mathbf{Q}^{n}-\mathbf{Q}^{n-1}}{\Delta t}+c \mathcal{V}^{n-2} \frac{\mathbf{Q}^{n-2}-\mathbf{Q}^{n-1}}{\Delta t}+d \mathcal{V}^{n-3} \frac{\mathbf{Q}^{n-3}-\mathbf{Q}^{n-1}}{\Delta t}+\mathbf{R}^{n}+R_{G C L}^{n} \mathbf{Q}^{n-1}=0
$$

Since the morphing grid formulation includes static meshes and rigid motion as special cases, the derivation is provided only for this formulation. Taking into account that $\mathbf{R}^{n}$ and $\mathbf{R}_{G C L}^{n}$ are dependent on $\mathbf{X}^{n-2}$ and $\mathbf{X}^{n-3}$, the procedure applied to the BDF1 scheme may also be used to derive the following adjoint equations for the flowfield:

for $3 \leq n \leq N$ :

$$
\begin{gathered}
\frac{a}{\Delta t}\left(\mathcal{V}^{n} \boldsymbol{\Lambda}_{f}^{n}-\mathcal{V}^{n+1} \boldsymbol{\Lambda}_{f}^{n+1}\right)+\frac{c}{\Delta t}\left(\mathcal{V}^{n} \boldsymbol{\Lambda}_{f}^{n+2}-\mathcal{V}^{n-1} \boldsymbol{\Lambda}_{f}^{n+1}\right)+\frac{d}{\Delta t}\left(\mathcal{V}^{n} \boldsymbol{\Lambda}_{f}^{n+3}-\mathcal{V}^{n-2} \boldsymbol{\Lambda}_{f}^{n+1}\right) \\
+\left[\frac{\partial \mathbf{R}^{n}}{\partial \mathbf{Q}^{n}}\right]^{T} \boldsymbol{\Lambda}_{f}^{n}+\mathbf{R}_{G C L}^{n+1} \mathbf{\Lambda}_{f}^{n+1}=-\left[\frac{\partial f^{n}}{\partial \mathbf{Q}^{n}}\right]^{T},
\end{gathered}
$$

where $\Lambda^{N+1}=\Lambda^{N+2}=\Lambda^{N+3}=0$;

for $n=2$ :

$$
\begin{aligned}
\frac{1}{\Delta t}\left(\frac{3}{2} \mathcal{V}^{2} \boldsymbol{\Lambda}_{f}^{2}-\right. & \left.a \mathcal{V}^{3} \boldsymbol{\Lambda}_{f}^{3}\right)+\frac{c}{\Delta t}\left(\mathcal{V}^{2} \boldsymbol{\Lambda}_{f}^{4}-\mathcal{V}^{1} \boldsymbol{\Lambda}_{f}^{3}\right)+\frac{d}{\Delta t}\left(\mathcal{V}^{2} \boldsymbol{\Lambda}_{f}^{5}-\mathcal{V}^{i n} \boldsymbol{\Lambda}_{f}^{3}\right) \\
& +\left[\frac{\partial \mathbf{R}^{2}}{\partial \mathbf{Q}^{2}}\right]^{T} \boldsymbol{\Lambda}_{f}^{2}+\mathbf{R}_{G C L}^{3} \boldsymbol{\Lambda}_{f}^{3}=-\left[\frac{\partial f^{2}}{\partial \mathbf{Q}^{2}}\right]^{T} ;
\end{aligned}
$$

for $n=1$ :

and for initial conditions:

$$
\begin{gathered}
\frac{1}{\Delta t}\left(\mathcal{V}^{1} \boldsymbol{\Lambda}_{f}^{1}-\frac{3}{2} \mathcal{V}^{2} \boldsymbol{\Lambda}_{f}^{2}\right)+\frac{1}{\Delta t}\left(c \mathcal{V}^{1} \boldsymbol{\Lambda}_{f}^{3}-\frac{1}{2} \mathcal{V}^{0} \boldsymbol{\Lambda}_{f}^{2}\right)+\frac{d}{\Delta t} \mathcal{V}^{1} \boldsymbol{\Lambda}_{f}^{4} \\
+\left[\frac{\partial \mathbf{R}^{1}}{\partial \mathbf{Q}^{1}}\right]^{T} \boldsymbol{\Lambda}_{f}^{1}+\mathbf{R}_{G C L}^{2} \boldsymbol{\Lambda}_{f}^{2}=-\left[\frac{\partial f^{1}}{\partial \mathbf{Q}^{1}}\right]^{T} ;
\end{gathered}
$$

$$
-\frac{1}{\Delta t} \mathcal{V}^{1} \boldsymbol{\Lambda}_{f}^{1}+\frac{1}{2 \Delta t} \mathcal{V}^{0} \boldsymbol{\Lambda}_{f}^{2}+\frac{d}{\Delta t} \mathcal{V}^{0} \boldsymbol{\Lambda}_{f}^{3}+\left[\frac{\partial \mathbf{R}^{i n}}{\partial \mathbf{Q}^{0}}\right]^{T} \boldsymbol{\Lambda}_{f}^{0}+\mathbf{R}_{G C L}^{1} \boldsymbol{\Lambda}_{f}^{1}=-\left[\frac{\partial f^{0}}{\partial \mathbf{Q}^{0}}\right]^{T}
$$


The corresponding mesh adjoint equations are obtained as follows. Assuming $\mathbf{R}^{N+1}=\mathbf{R}^{N+2}=\mathbf{R}^{N+3}=0$ and $\mathbf{R}_{G C L}^{N+1}=\mathbf{R}_{G C L}^{N+2}=\mathbf{R}_{G C L}^{N+3}=0$ :

$$
\begin{aligned}
& {\left[\mathcal{K}^{0}\right]^{T} \boldsymbol{\Lambda}_{g}^{n}=\left[\frac{\partial f^{n}}{\partial \mathbf{X}^{n}}\right]^{T}} \\
& +\left[a\left(\frac{\partial \mathcal{V}^{n}}{\partial \mathbf{X}^{n}} \frac{\mathbf{Q}^{n}-\mathbf{Q}^{n-1}}{\Delta t}\right)^{T} \boldsymbol{\Lambda}_{f}^{n}+c\left(\frac{\partial \mathcal{V}^{n}}{\partial \mathbf{X}^{n}} \frac{\mathbf{Q}^{n}-\mathbf{Q}^{n+1}}{\Delta t}\right)^{T} \boldsymbol{\Lambda}_{f}^{n+2}+d\left(\frac{\partial \mathcal{V}^{n}}{\partial \mathbf{X}^{n}} \frac{\mathbf{Q}^{n}-\mathbf{Q}^{n+2}}{\Delta t}\right)^{T} \boldsymbol{\Lambda}_{f}^{n+3}\right] \\
& +\sum_{k=0}^{3}\left\{\left[\frac{\partial \mathbf{R}^{n+k}}{\partial \mathbf{X}^{n}}+\frac{\partial \boldsymbol{R}_{G C L}^{n+k}}{\partial \mathbf{X}^{n}} \mathbf{Q}^{n+k-1}\right]^{T} \boldsymbol{\Lambda}_{f}^{n+k}\right\}, \text { for } 3 \leq n \leq N \\
& {\left[\mathcal{K}^{0}\right]^{T} \Lambda_{g}^{2}=\left[\frac{\partial f^{2}}{\partial \mathbf{X}^{2}}\right]^{T}} \\
& +\left[\frac{3}{2}\left(\frac{\partial \mathcal{V}^{2}}{\partial \mathbf{X}^{2}} \frac{\mathbf{Q}^{2}-\mathbf{Q}^{1}}{\Delta t}\right)^{T} \mathbf{\Lambda}_{f}^{2}+c\left(\frac{\partial \mathcal{V}^{2}}{\partial \mathbf{X}^{2}} \frac{\mathbf{Q}^{2}-\mathbf{Q}^{3}}{\Delta t}\right)^{T} \boldsymbol{\Lambda}_{f}^{4}+d\left(\frac{\partial \mathcal{V}^{2}}{\partial \mathbf{X}^{2}} \frac{\mathbf{Q}^{2}-\mathbf{Q}^{4}}{\Delta t}\right)^{T} \boldsymbol{\Lambda}_{f}^{5}\right] \\
& +\sum_{k=0}^{3}\left\{\left[\frac{\partial \mathbf{R}^{2+k}}{\partial \mathbf{X}^{2}}+\frac{\partial \boldsymbol{R}_{G C L}^{2+k}}{\partial \mathbf{X}^{2}} \mathbf{Q}^{1+k}\right]^{T} \boldsymbol{\Lambda}_{f}^{2+k}\right\} \text {, for } n=2 \\
& {\left[\mathcal{K}^{0}\right]^{T} \boldsymbol{\Lambda}_{g}^{1}=\left[\frac{\partial f^{1}}{\partial \mathbf{X}^{1}}\right]^{T}} \\
& +\left[\left(\frac{\partial \mathcal{V}^{1}}{\partial \mathbf{X}^{1}} \frac{\mathbf{Q}^{1}-\mathbf{Q}^{0}}{\Delta t}\right)^{T} \boldsymbol{\Lambda}_{f}^{1}+c\left(\frac{\partial \mathcal{V}^{1}}{\partial \mathbf{X}^{1}} \frac{\mathbf{Q}^{1}-\mathbf{Q}^{2}}{\Delta t}\right)^{T} \boldsymbol{\Lambda}_{f}^{3}+d\left(\frac{\partial \mathcal{V}^{1}}{\partial \mathbf{X}^{1}} \frac{\mathbf{Q}^{1}-\mathbf{Q}^{3}}{\Delta t}\right)^{T} \mathbf{\Lambda}_{f}^{4}\right] \\
& +\sum_{k=0}^{3}\left\{\left[\frac{\partial \mathbf{R}^{1+k}}{\partial \mathbf{X}^{1}}+\frac{\partial \mathcal{R}_{G C L}^{1+k}}{\partial \mathbf{X}^{1}} \mathbf{Q}^{k}\right]^{T} \Lambda_{f}^{1+k}\right\}, \text { for } n=1 ;
\end{aligned}
$$

and for initial conditions, $\mathbf{R}^{\text {in }} \equiv \mathbf{Q}^{\infty}-\mathbf{Q}^{0}$ :

$$
\begin{gathered}
{\left[\mathcal{K}^{0}+\frac{\partial \mathcal{K}^{0}}{\partial \mathbf{X}^{0}} \mathbf{X}^{0}\right]^{T} \Lambda_{g}^{0}=\left[\frac{\partial f^{0}}{\partial \mathbf{X}^{0}}\right]^{T}+\left[\frac{1}{2}\left(\frac{\partial \mathcal{V}^{0}}{\partial \mathbf{X}^{0}} \frac{\mathbf{Q}^{0}-\mathbf{Q}^{1}}{\Delta t}\right)^{T} \Lambda_{f}^{2}+d\left(\frac{\partial \mathcal{V}^{0}}{\partial \mathbf{X}^{0}} \frac{\mathbf{Q}^{0}-\mathbf{Q}^{2}}{\Delta t}\right)^{T} \boldsymbol{\Lambda}_{f}^{3}\right]} \\
+\left[\frac{\partial \mathbf{Q}^{\infty}}{\partial \mathbf{X}^{0}}\right]^{T} \Lambda_{f}^{0}+\sum_{k=1}^{3}\left\{\left[\frac{\partial \mathbf{R}^{k}}{\partial \mathbf{X}^{0}}+\frac{\partial \mathcal{R}_{G C L}^{k}}{\partial \mathbf{X}^{0}} \mathbf{Q}^{k-1}\right]^{T} \Lambda_{f}^{k}\right\}-\sum_{n=1}^{N}\left[\frac{\partial \mathcal{K}^{0}}{\partial \mathbf{X}^{0}} \mathbf{X}^{n}\right]^{T} \Lambda_{g}^{n}
\end{gathered}
$$

The sensitivity derivative for the higher-order BDF schemes is evaluated using Eq. (23). 
Table 1. Results for static grid test case. "A" denotes adjoint result, "C" denotes complex-variable result.

\begin{tabular}{ccccc}
\hline \hline Design Variable & \multirow{2}{*}{ BDF1 } & BDF2 & BDF3 & BDF2 ${ }_{\text {opt }}$ \\
\hline Angle of & A: 0.004249541855867 & A: 0.003734353591935 & A: 0.003687377975335 & A: 0.003708754474661 \\
Attack & C: 0.004249541855867 & C: 0.003734353591935 & C: 0.003687377975335 & C: 0.003708754474661 \\
& & & & \\
Camber & A: 0.010713047647152 & A: 0.013701437304586 & A: 0.014574974114575 & A: 0.014145698047604 \\
& C: 0.010713047647155 & C: 0.013701437304586 & C: 0.014574974114577 & C: 0.014145698047602 \\
\hline \hline
\end{tabular}

Table 2. Results for rigidly moving grid. "A" denotes adjoint result, "C" denotes complex-variable result.

\begin{tabular}{ccccc}
\hline \hline Design Variable & BDF1 & BDF2 & BDF3 & BDF2 opt \\
\hline $\begin{array}{c}\text { Angle of } \\
\text { Attack }\end{array}$ & A: 0.004713138571667 & A: 0.004293218571759 & A: 0.004245785984455 & A: 0.004267302756747 \\
& C: 0.004713138571667 & C: 0.004293218571759 & C: 0.004245785984455 & C: 0.004267302756681 \\
Pitching & A: -0.403740396501207 & A: -0.527819225717431 & A: -0.52983335959555333 & A: -0.5288894917963836 \\
Frequency & C: -0.403740396501207 & C: -0.527819225717432 & C: -0.529833595955533 & C: -0.528894917963837 \\
& A: 0.011630821689945 & A: 0.013925365539211 & A: 0.0142912283344440 & A: 0.014071544549783 \\
Camber & C: 0.011630821689944 & C: 0.013925365539206 & C: 0.014291228334428 & C: 0.014071544549783 \\
\hline \hline
\end{tabular}

Table 3. Results for morphing grid. "A" denotes adjoint result, "C" denotes complex-variable result.

\begin{tabular}{ccccc}
\hline \hline Design Variable & BDF1 & BDF2 & BDF3 & BDF2 ${ }_{\text {opt }}$ \\
\hline Angle of & A: 0.004713528355526 & A: 0.004298221887378 & A: 0.004250753632738 & A: 0.004272205860974 \\
Attack & C: 0.004713528355526 & C: 0.004298221887378 & C: 0.004250753632738 & C: 0.004272205860974 \\
& & & & \\
Pitching & A: -0.403961428430834 & A: -0.528263525075847 & A: -0.530205775809711 & A: -0.529295291075346 \\
Frequency & C: -0.403961428430834 & C: -0.528263525075847 & C: -0.530205775809710 & C: -0.529295291075346 \\
& & & & \\
Camber & A: 0.011680362720549 & A: 0.013922237526691 & A: 0.014268675858452 & A: 0.014055458873064 \\
& C: 0.011680362720548 & C: 0.013922237526686 & C: 0.014268675858435 & C: 0.014055458873058 \\
\hline \hline
\end{tabular}

Table 4. Coefficients for higher-order BDF schemes.

\begin{tabular}{ccccc}
\hline \hline Scheme & $a$ & $b$ & $c$ & $d$ \\
\hline BDF2 & $3 / 2$ & -2 & $1 / 2$ & 0 \\
BDF3 & $11 / 6$ & -3 & $3 / 2$ & $-1 / 3$ \\
$\mathrm{BDF}_{\mathrm{opt}}$ & $5.08 / 3$ & -2.58 & 1.08 & $-0.58 / 3$ \\
\hline \hline
\end{tabular}




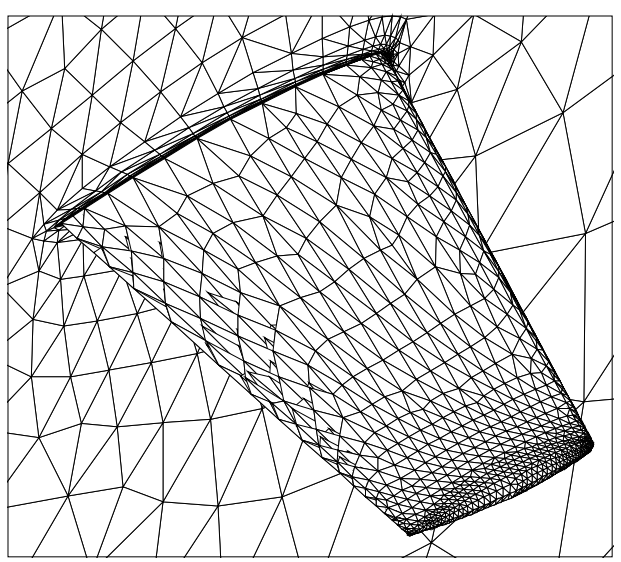

Figure 1. Surface grid for ONERA M6 wing.

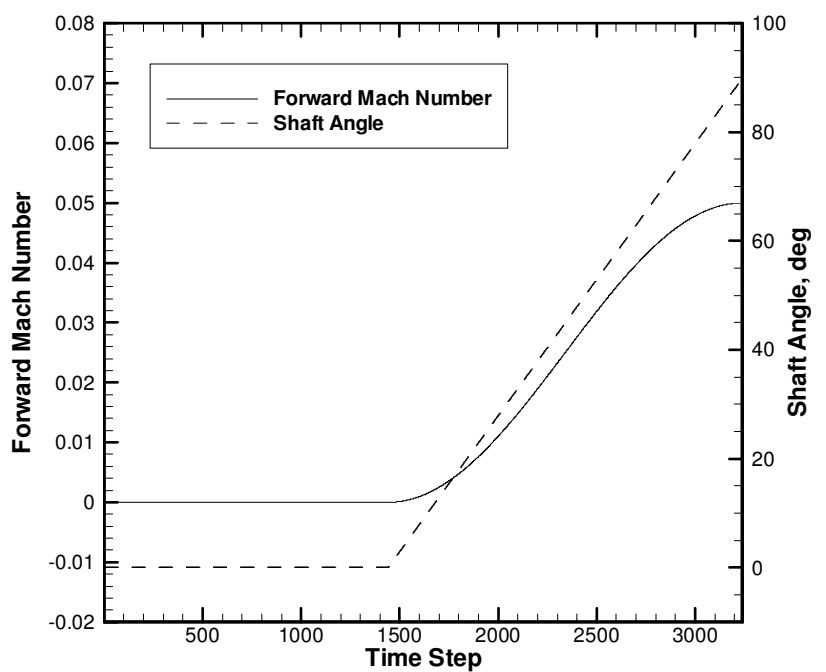

Figure 2. Forward Mach number and shaft angle schedule for TRAM rotor simulation.

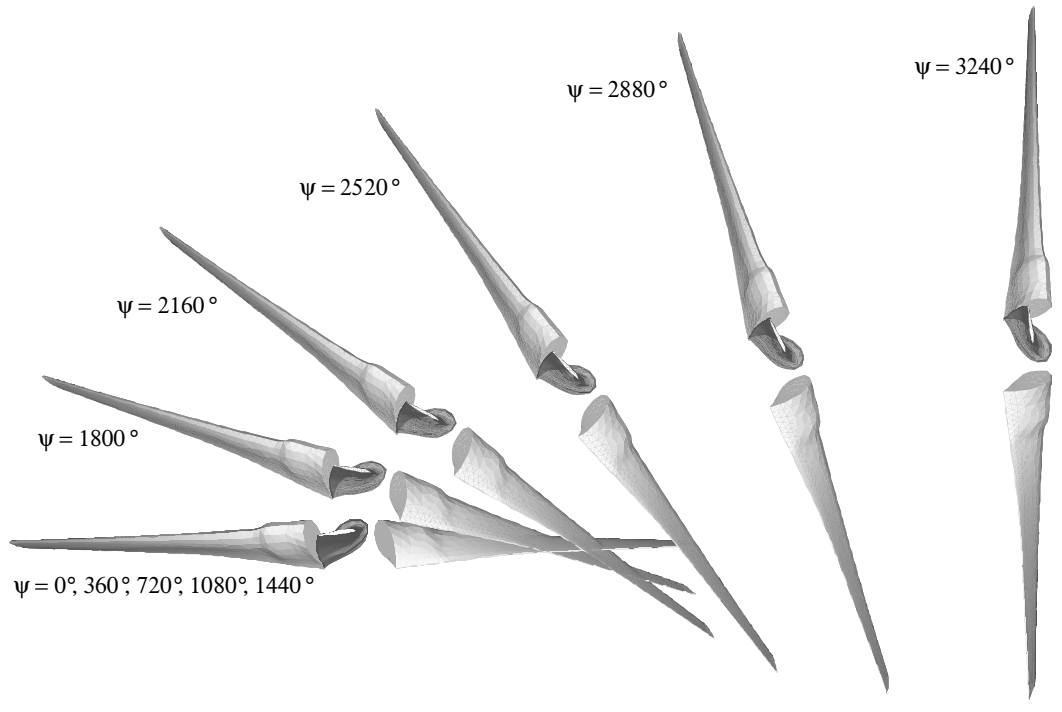

Figure 3. View of TRAM rotor motion. 


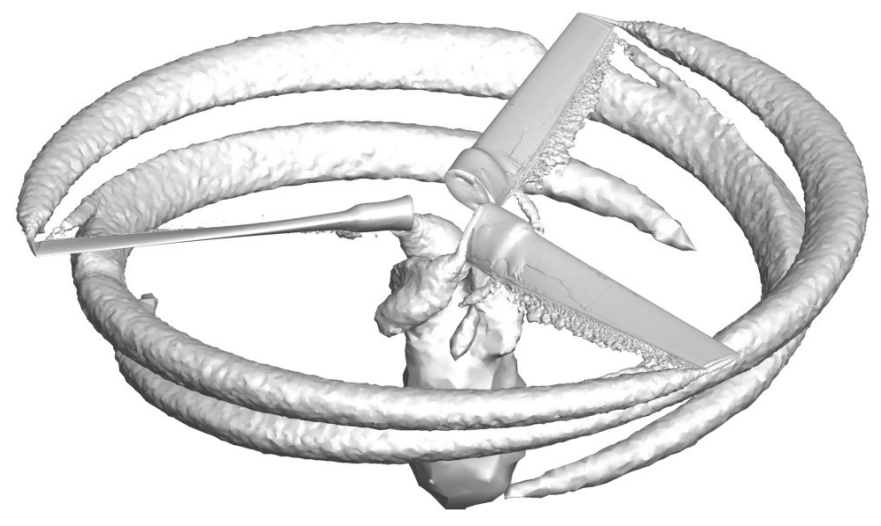

Figure 4. Isosurface of Q criterion for TRAM rotor at $\Psi=1440^{\circ}$.

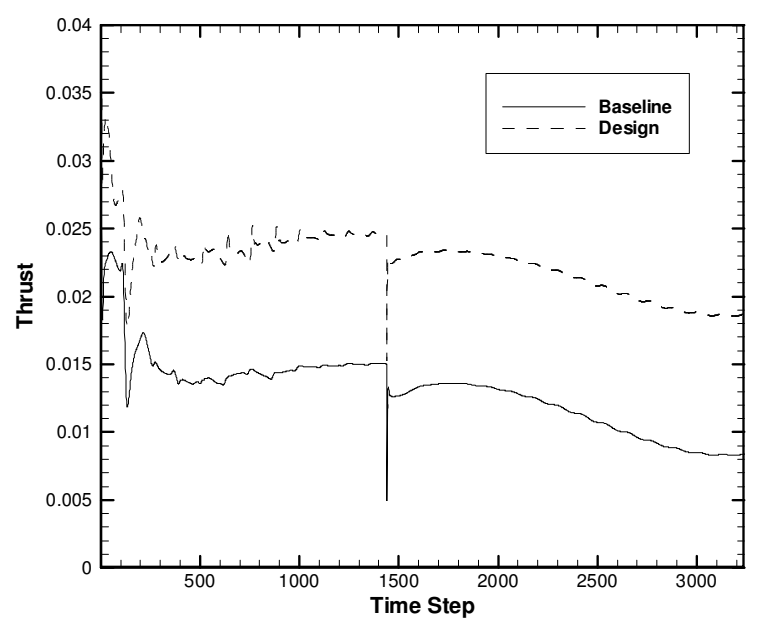

Figure 5. Thrust for TRAM rotor before and after design optimization.

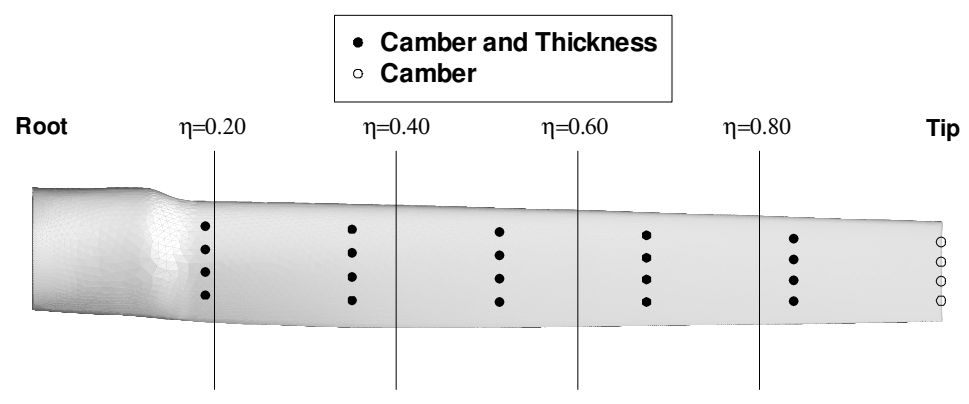

Figure 6. Spanwise blade and design variable locations for TRAM rotor. 


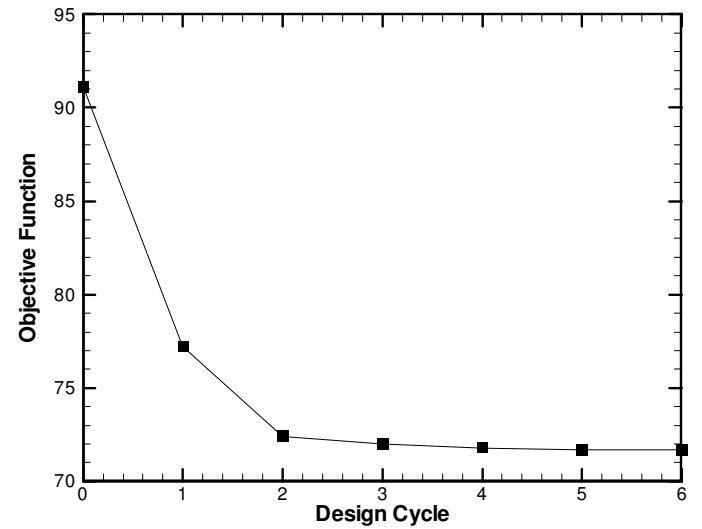

Figure 7. Objective function history for TRAM rotor.

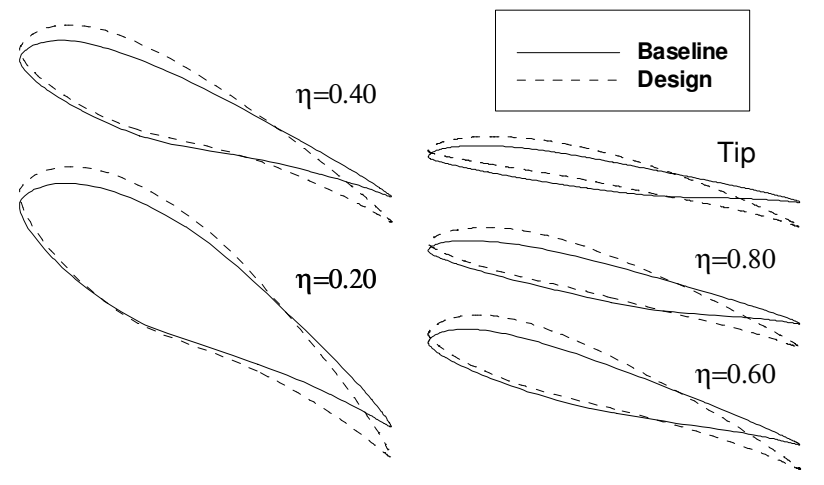

Figure 8. Spanwise blade cross-sections before and after optimization of TRAM rotor.

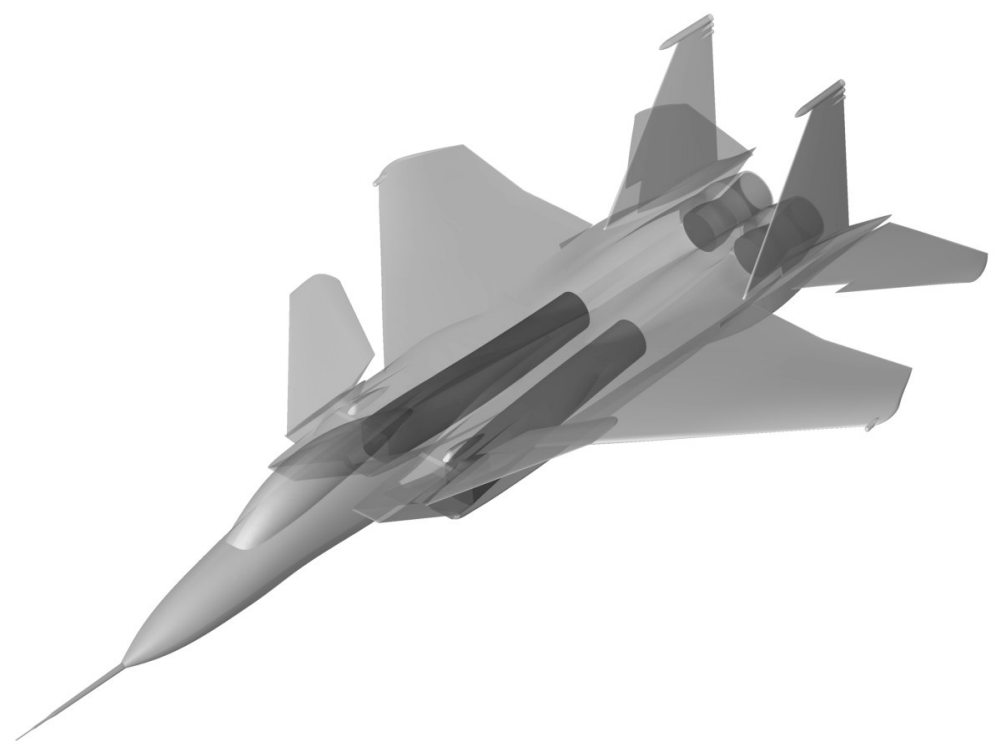

Figure 9. Modified F-15 with engine duct geometry. 


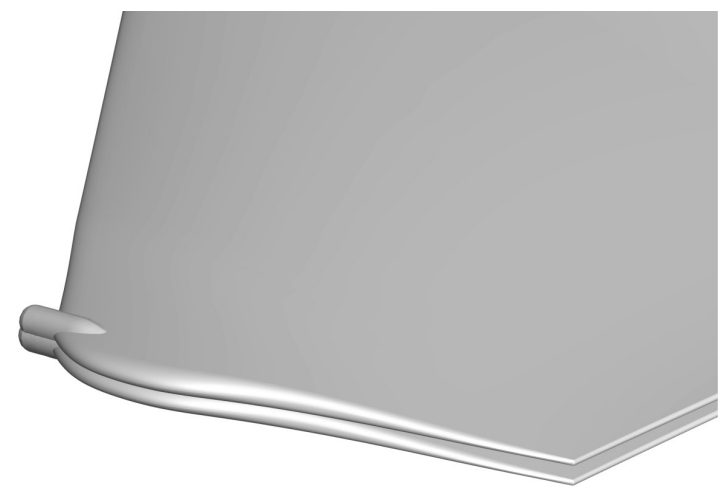

Figure 10. Range of prescribed motion for modified F-15 wingtip.

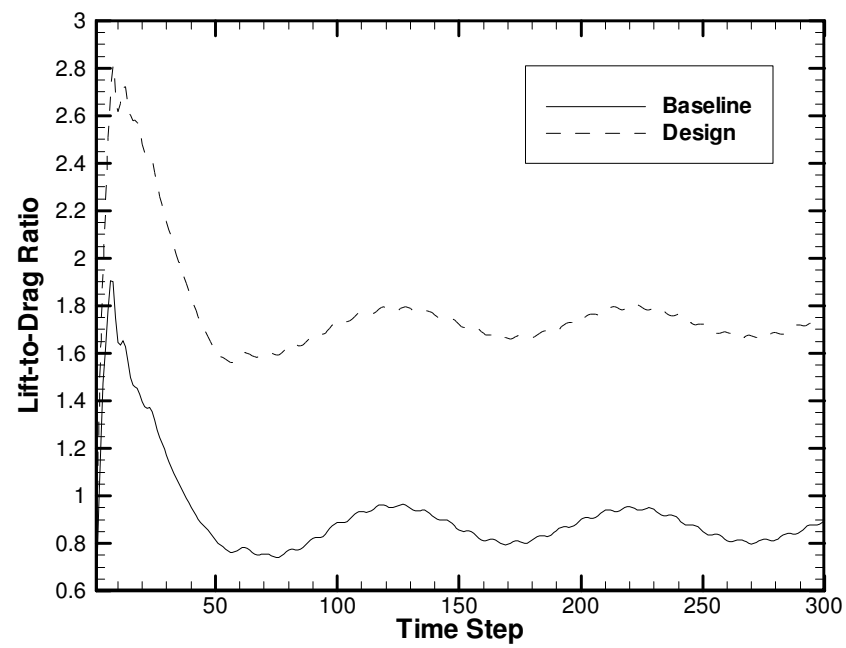

Figure 11. Lift-to-drag ratio for modified F-15 before and after design optimization.

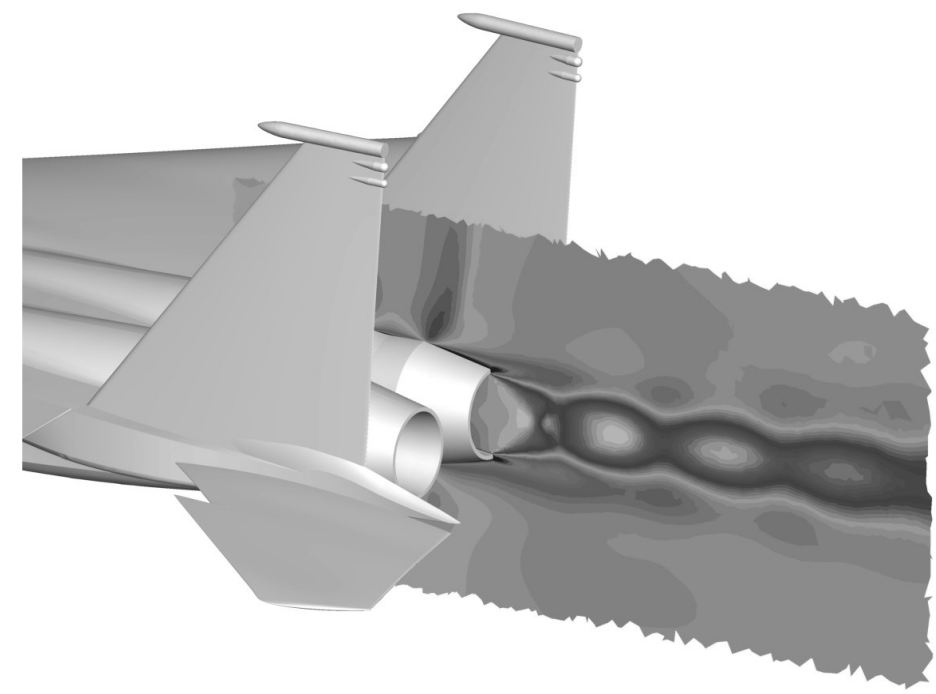

Figure 12. Cross-section of engine plume contours for modified F-15. 


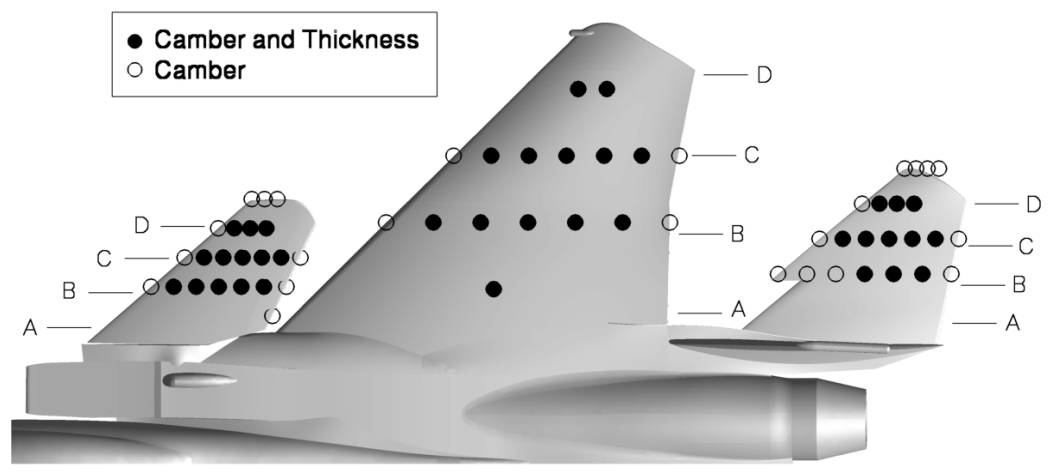

Figure 13. Spanwise and design variable locations for modified F-15.

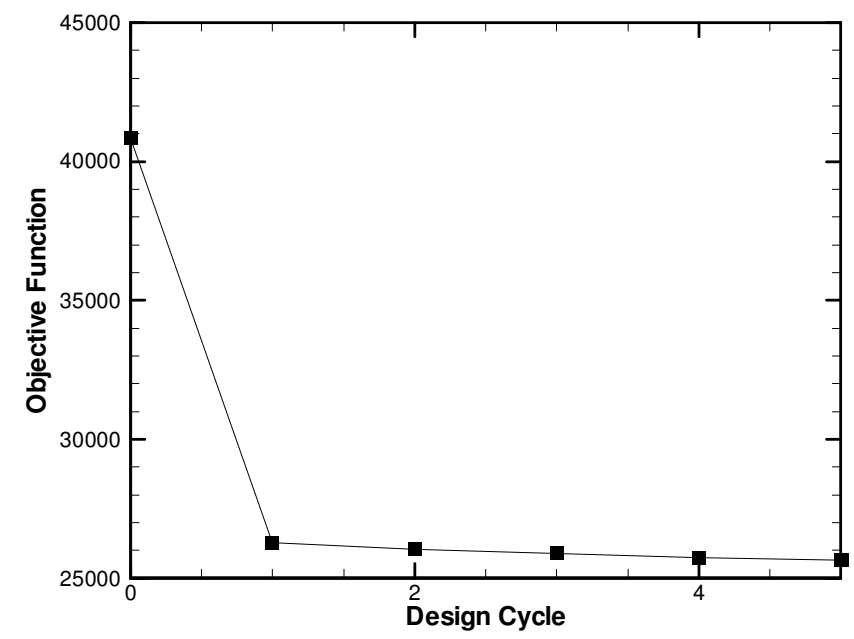

Figure 14. Objective function history for modified F-15.

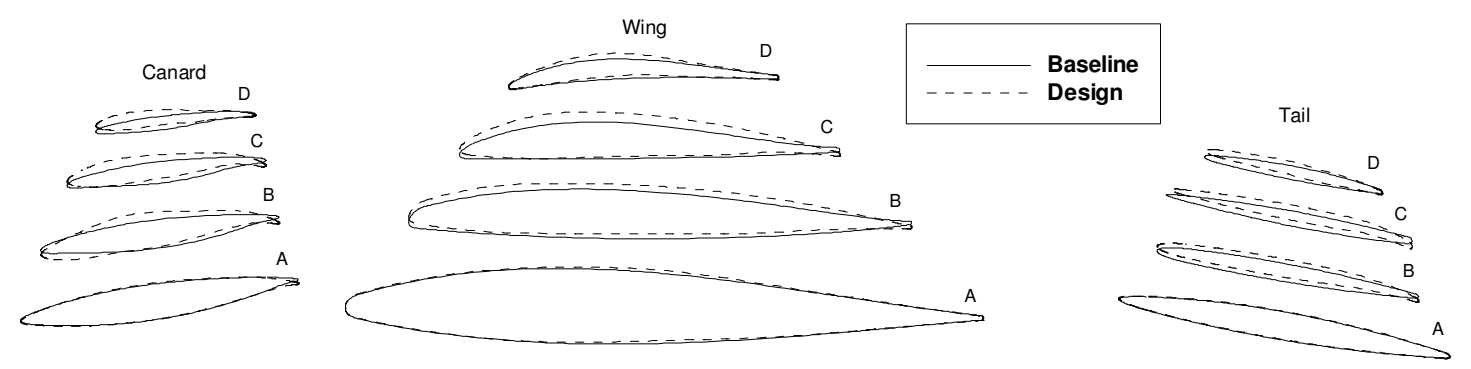

Figure 15. Canard, wing, and tail cross-sections before and after optimization of modified F-15. 